\title{
Shared Molecular Mechanisms between Alzheimer's Disease and Periodontitis Revealed by Transcriptomic Analysis
}

\author{
Jieqi Jin $\mathbb{D}^{1},{ }^{1}$ Mengkai Guang $\mathbb{D}^{2},{ }^{2}$ Anthony Chukwunonso Ogbuehi $\mathbb{D}^{3}{ }^{3} \operatorname{Simin} \mathrm{Li}\left(\mathbb{D},{ }^{4}\right.$ \\ Kai Zhang $\mathbb{D}^{2}{ }^{2}$ Yihong Ma $\mathbb{D}^{5},{ }^{5}$ Aneesha Acharya $\mathbb{D}^{6},{ }^{6}$ Bihan Guo $\mathbb{D}^{7},{ }^{7}$ Zongwu Peng $\mathbb{D}^{7},{ }^{7}$ \\ Xiangqiong Liu $\mathbb{D}^{8},{ }^{8}$ Yupei Deng $\mathbb{D}^{8},{ }^{8}$ Zhaobi Fang $\mathbb{D}^{9},{ }^{9}$ Xiongjie Zhu $\mathbb{D}^{\text {, }}$, Shiting Hua $\mathbb{D}^{9}$, \\ Cong Li $\mathbb{D}^{, 9}$ Rainer Haak $\mathbb{D}^{4}{ }^{4}$ Dirk Ziebolz ${ }^{(D},{ }^{4}$ Gerhard Schmalz ${ }^{\mathbb{D}},{ }^{4}$ Lei Liu $\mathbb{D}^{10}{ }^{10}$ \\ Baohua Xu $\mathbb{D},{ }^{2}$ and Xiaofeng Huang $\mathbb{D}^{1}$ \\ ${ }^{1}$ Department of Stomatology, Beijing Friendship Hospital, Capital Medical University, Beijing 100050, China \\ ${ }^{2}$ Department of Stomatology, China-Japan Friendship Hospital, Beijing 100029, China \\ ${ }^{3}$ Department of Physics, University of Münster, Wilhelm-Klemm-Str. 9, 48149 Münster, Germany \\ ${ }^{4}$ Department of Cariology, Endodontology and Periodontology, University Leipzig, Liebigstr. 12, Leipzig 04103, Germany \\ ${ }^{5}$ Department of Neurology, Graduate School of Medical Sciences, Faculty of Life Sciences, Kumamoto University, Kumamoto, Japan \\ ${ }^{6}$ Dr. D Y Patil Dental College and Hospital, Dr D Y Patil Vidyapeeth, Pimpri, Pune, India \\ ${ }^{7}$ Faculty of Electrical Engineering, Information Technology, and Physics, University Braunschweig, Hans-Sommer-Str. 66, \\ Braunschweig 38106, Germany \\ ${ }^{8}$ Laboratory of Molecular Cell Biology, Beijing Tibetan Hospital, China Tibetology Research Center, 218 Anwaixiaoguanbeili Street, \\ Chaoyang, Beijing 100029, China \\ ${ }^{9}$ Zhujiang Hospital, Southern Medical University, Guangzhou 510282, China \\ ${ }^{10}$ Department of Neurology, Shandong Provincial Third Hospital, Cheeloo College of Medicine, Shandong University, Jinan, \\ 10091 Shandong Province, China
}

Correspondence should be addressed to Baohua Xu; zrkqxbh@163.com

Received 18 December 2020; Revised 20 February 2021; Accepted 9 March 2021; Published 1 April 2021

Academic Editor: Juan Yang

Copyright (c) 2021 Jieqi Jin et al. This is an open access article distributed under the Creative Commons Attribution License, which permits unrestricted use, distribution, and reproduction in any medium, provided the original work is properly cited.

Objective. To investigate the genetic crosstalk mechanisms that link periodontitis and Alzheimer's disease (AD). Background. Periodontitis, a common oral infectious disease, is associated with Alzheimer's disease (AD) and considered a putative contributory factor to its progression. However, a comprehensive investigation of potential shared genetic mechanisms between these diseases has not yet been reported. Methods. Gene expression datasets related to periodontitis were downloaded from the Gene Expression Omnibus (GEO) database, and differential expression analysis was performed to identify differentially expressed genes (DEGs). Genes associated with AD were downloaded from the DisGeNET database. Overlapping genes among the DEGs in periodontitis and the AD-related genes were defined as crosstalk genes between periodontitis and AD. The Boruta algorithm was applied to perform feature selection from these crosstalk genes, and representative crosstalk genes were thus obtained. In addition, a support vector machine (SVM) model was constructed by using the scikit-learn algorithm in Python. Next, the crosstalk gene-TF network and crosstalk gene-DEP (differentially expressed pathway) network were each constructed. As a final step, shared genes among the crosstalk genes and periodontitis-related genes in DisGeNET were identified and denoted as the core crosstalk genes. Results. Four datasets (GSE23586, GSE16134, GSE10334, and GSE79705) pertaining to periodontitis were included in the analysis. A total of 48 representative crosstalk genes were identified by using the Boruta algorithm. Three TFs (FOS, MEF2C, and USF2) and several pathways (i.e., JAK-STAT, MAPK, NF-kappa B, and natural killer cell-mediated cytotoxicity) were identified as regulators of these crosstalk genes. Among these 48 crosstalk genes and the chronic periodontitis-related genes in DisGeNET, C4A, C4B, CXCL12, FCGR3A, IL1B, and MMP3 were shared and identified as the most pivotal candidate links between periodontitis and AD. Conclusions. Exploration of available transcriptomic datasets revealed C4A, C4B, CXCL12, FCGR3A, IL1B, and MMP3 as the top candidate molecular linkage genes between periodontitis and AD. 


\section{Introduction}

An association between periodontitis and Alzheimer's disease $(\mathrm{AD})$ has been demonstrated, and periodontitis reportedly confers risk for the incidence and progression of Alzheimer's disease (AD) [1, 2]. Alzheimer's disease is a neurodegenerative disease characterized by the formation of amyloid- $\beta$ peptide $(\mathrm{A} \beta \mathrm{P})$ plaques and intraneuronal neurofibrillary tangles (NFTs), which drives neuroinflammation in the brain [3]. Periodontitis, a chronic, immunoinflammatory disease affecting supporting structures of teeth, is multifactorial in nature, driven by polymicrobial dysbiosis and unfavorable shifts in the plaque biofilm composition, which disrupts the host-microbial homeostasis $[4,5]$. Inflammation is considered the key connecting link between both these diseases [6]. Two purported mechanistic links have been highlighted. First, proinflammatory mediators including specific cytokines or chemokines in the periodontal milieu that enter systemic circulation impose a systemic inflammatory burden, propagating inflammatory responses by microglial cells in the brain [6]. Second, periodontal pathogens may directly enter the brain via blood circulation or peripheral nerves, as evidenced by the discovery of the keystone periodontal pathogen Porphyromonas gingivalis (Pg) in AD patients' brains $[7,8]$.

At the same time, genetic susceptibility and gene dysregulation have been identified in the context of both $\mathrm{AD}$ and periodontitis. In $\mathrm{AD}$, frequently reported dysregulated genes include amyloid-beta precursor protein (APP), presenilin 1 (PSEN1), and presenilin 2 (PSEN2), apolipoproteins, and lipid homeostasis, genes involved in endocytosis, and membrane-spanning 4 (MS4) family [9]. In periodontitis, aberrant genes highlighted include interleukin-1, interleukin-6, interleukin-10, transforming growth factor-beta (TGF- $\beta$ ), tumor necrosis factor- $\alpha$ (TNF- $\alpha$ ), interferongamma (IFN- $\gamma$ ), and matrix metalloproteinases (MMPs) among others [10]. It is plausible that gene dysregulation in periodontitis could contribute to its association with $\mathrm{AD}$, and crosstalk genes may biologically link $\mathrm{AD}$ and periodontitis serving as either shared susceptibility factors or molecular links.

Here, we designed a bioinformatic study of existing experimental datasets to understand putative molecular links between periodontitis and Alzheimer's disease by identifying crosstalk genes, transcription factors, and signaling pathways involved in both disorders. The molecular mechanisms identified through this approach could suggest potential therapeutic targets particularly relevant to drug development and personalized medicine approaches.

\section{Materials and Methods}

2.1. Study Design. Figure 1 depicts a flowchart outlining the study workflow. DEGs dysregulated in periodontitis- and AD-related genes were obtained from the GEO database and DisGeNET database, respectively. Crosstalk genes linking periodontitis and $\mathrm{AD}$ were identified as the $\mathrm{AD}$-related genes that overlapped with significantly up- or downregulated DEGs in periodontitis. Thereafter, feature selection from the crosstalk genes was performed using a conventional recursive feature elimination (RFE) algorithm and the Boruta algorithm. The crosstalk genes obtained by feature selection were used to construct two networks to identify the transcription factors and the differentially expressed pathways that target these crosstalk genes. In the next step, "core" crosstalk genes were identified as the crosstalk genes obtained by feature selection that were overlapping with chronic periodontitis-related genes in the DisGeNET database.

2.2. Procurement of Periodontitis-Related Datasets. Samplematched whole-genome gene expression datasets from periodontitis were sourced and downloaded from the NCBI Gene Expression Omnibus (GEO). The eligibility criteria for these datasets were as follows: datasets that included established periodontitis samples as the experimental group and healthy gingival samples as the control group, where periodontitis was defined based in accordance with the case definition presented in the 2017 World Workshop: (1) interdental CAL detectable at $\geq 2$ nonadjacent teeth or (2) buccal or oral CAL $\geq 3 \mathrm{~mm}$ with pocketing $>3 \mathrm{~mm}$ detectable at $\geq 2$ teeth [11].

2.3. Differential Gene Expression Analysis. Differential gene expression analysis of periodontitis-related datasets was carried out using the Linear Models for Microarray (limma) package [12] in the $\mathrm{R}$ project (version 3.0.1, http://www.rproject.org/) [13]. Three such datasets, GSE23586, GSE16134, and GSE10334, were sourced and analyzed. Genes with $p$ value $<0.05$ and $\mid \operatorname{logFC}$ ( fold change) $\mid \geq 1$ were regarded as significant differentially expressed genes (DEGs). For another dataset, GSE79705, the screening range of DEGs was broadened by extending the thresholds and settings to $p$ value $<0.05$ and $|\log \mathrm{FC}|>0$ as DEGs.

Next, a Venn diagram (http://bioinformatics.psb.ugent .be/webtools/Venn/) was drawn to identify shared genes within the DEGs identified from the four datasets. The common up/downregulated DEGs in four datasets were used for the following analyses, and DEGs that were not common to all the datasets were excluded.

2.4. Functional Enrichment Analysis. Functional enrichment analysis of up/downregulated periodontitis-related DEGs was performed with DAVID (Database for Annotation, Visualization and Integrated Discovery, v6.8) [14, 15]. $p<0.05$ was set as the threshold. GO (Gene Ontology) and pathway enrichment analysis of the identified DEGs was performed $[16,17]$.

2.5. Construction of the Protein-Protein Interaction Network of Periodontitis-Related DEGs. 280,826 PPI (protein-protein interaction) pairs including 19,610 genes were downloaded from HPRD (Human Protein Reference Database) [18], BioGRID (Biological General Repository for Interaction Datasets) [19], DIP (Database of Interacting Proteins) [20], MINT (Molecular INTeraction database) [21], PINA (Protein Interaction Network Analysis) [22], InnateDB (a knowledge resource for innate immunity interactions and pathways) [23], and INstruct (3D protein interactome networks with structural resolution) [24]. The Cytoscape 


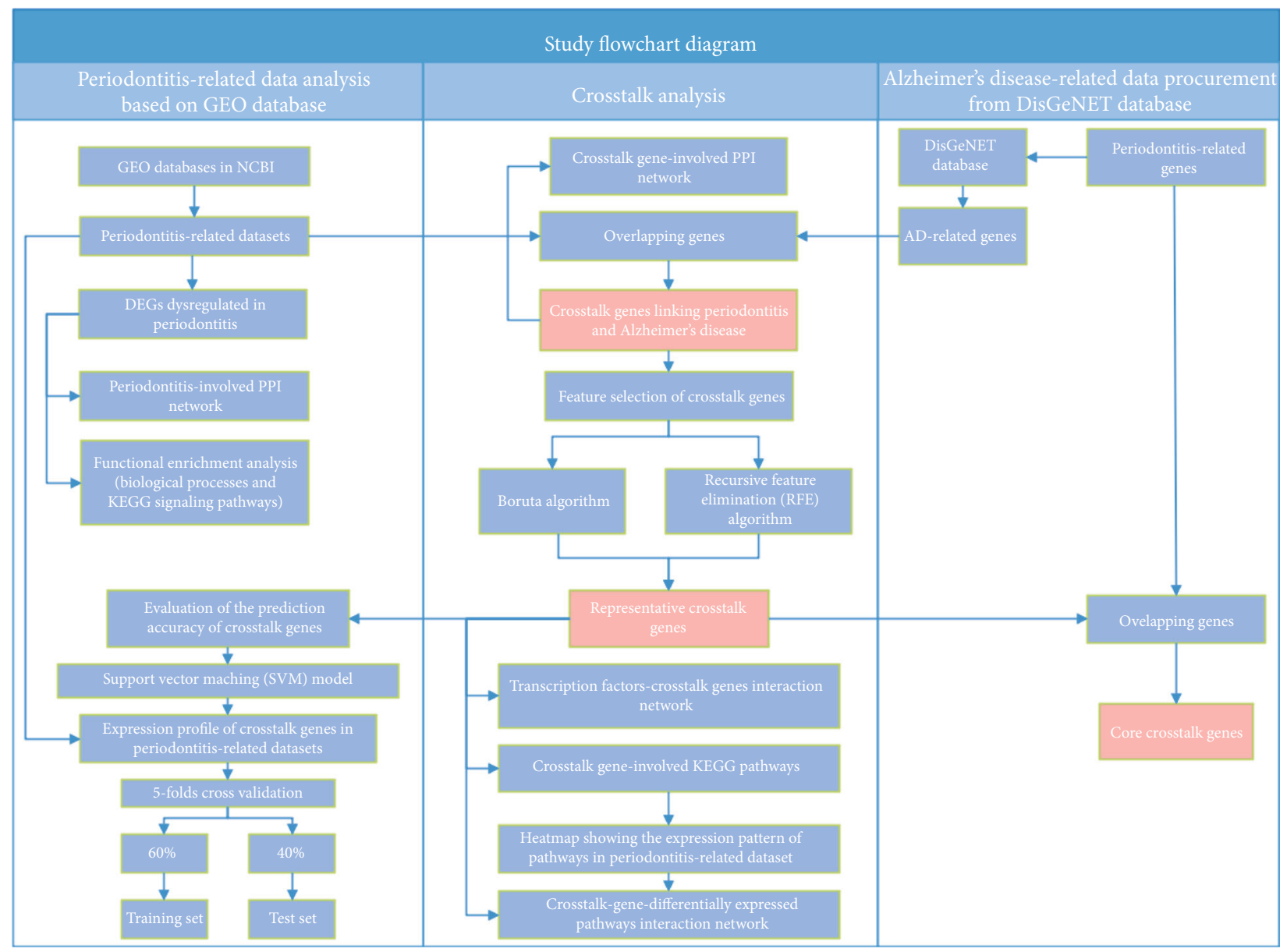

FIGURE 1: Flowchart depicting study workflow.

platform was used to visualize the network derived from PPI pairs of DEGs and conduct a topological analysis [25].

2.6. Identification of Crosstalk Genes and Construction of the Crosstalk Gene-Related PPI Network. AD-related genes were downloaded from the DisGeNET database [26]. The ADrelated genes that overlapped with the up- and downregulated periodontitis-related DEGs were identified. These overlapping genes were regarded as "crosstalk" genes linking AD and periodontitis and further used for constructing a crosstalk gene-related PPI network. The crosstalk gene-related PPI network consisted of four types of nodes, namely, (1) DEGs dysregulated in periodontitis (not related to AD), (2) crosstalk genes or AD-related genes which were also DEGs dysregulated in periodontitis, (3) AD-related genes (not dysregulated in periodontitis), and (4) other genes (neither related to $\mathrm{AD}$ nor dysregulated in periodontitis).

2.7. Feature Selection from Crosstalk Genes. Since GSE16134 had the largest sample size among the periodontitis-related datasets, it was used as the test set. The other three datasets (GSE23586, GSE10334, and GSE79705) were used as validation sets. Firstly, expression values of the crosstalk genes (identified in the previous step) from GSE16134 were used as input for the Boruta algorithm in the $\mathrm{R}$ project [27] and the conventional recursive feature elimination (RFE) algorithm [28] and feature selection was performed. Each gene was regarded as a feature.

2.8. Support Vector Machine (SVM) Modeling Using FeatureSelected Crosstalk Genes. The expression values in GSE16134 and GSE10334 were scale-standardized. Next, it was examined if the crosstalk genes obtained by feature selection were found in the four periodontitis-related datasets (i.e., GSE23586, GSE16134, GSE10334, and GSE79705). The gene expression values of these feature selection-obtained crosstalk genes were extracted from these datasets. If the number of expression profile genes in a certain dataset after extraction was lower than the number of feature selectionobtained crosstalk genes, the expression values of the missing genes were considered missing values and represented by the NA symbol. The missing values were processed by using the DMwR package [29] in $\mathrm{R}$, and the $K$-Nearest Neighbors (KNN) algorithm was used to impute these missing values (NA) in that dataset. By imputing these missing values, all the four periodontitis-related datasets presented all the feature selection-obtained crosstalk genes. Thereafter, the scikit-learn package [30] was used to perform a grid search, and the best hyperparameters of a support vector machine (SVM) model were found by using 5-fold cross-validation 
TABLe 1: Details of the included periodontitis-related GEO datasets.

\begin{tabular}{|c|c|c|c|c|c|c|}
\hline $\begin{array}{l}\text { Included four } \\
\text { datasets }\end{array}$ & $\begin{array}{c}\text { Type of } \\
\text { periodontitis }\end{array}$ & $\begin{array}{l}\text { Experimental } \\
\text { platform }\end{array}$ & $\begin{array}{l}\text { Number of } \\
\text { examined } \\
\text { genes }\end{array}$ & $\begin{array}{l}\text { Number of } \\
\text { inflamed } \\
\text { gingival } \\
\text { tissue samples }\end{array}$ & $\begin{array}{l}\text { Number of } \\
\text { healthy } \\
\text { control } \\
\text { samples }\end{array}$ & $\begin{array}{l}\text { Number of } \\
\text { total samples }\end{array}$ \\
\hline GSE23586 & $\begin{array}{l}3 \text { patients with severe } \\
\text { chronic periodontitis }\end{array}$ & GPL570 & 23,518 & 3 & 3 & 6 \\
\hline GSE16134 & $\begin{array}{l}120 \text { patients ( } 65 \text { with } \\
\text { chronic periodontitis } \\
\text { and } 55 \text { with aggressive } \\
\text { periodontitis) }\end{array}$ & GPL570 & 24,441 & 241 & 69 & 310 \\
\hline GSE10334 & $\begin{array}{c}63 \text { with chronic periodontitis } \\
\text { and } 27 \text { with aggressive } \\
\text { periodontitis }\end{array}$ & GPL570 & 24,441 & 183 & 64 & 247 \\
\hline GSE79705 & $\begin{array}{c}\text { Generalized aggressive } \\
\text { periodontitis (GAgP): } \\
n=4 \text {; chronic } \\
\text { periodontitis }(\mathrm{CP}): n=4\end{array}$ & GPL18734 & 19,305 & 8 & 4 & 12 \\
\hline
\end{tabular}

TABLE 2: The number of up/downregulated DEGs identified in the four periodontitis-related datasets GSE23586, GSE16134, GSE10334, and GSE79705 at thresholds set for defining DEGs.

\begin{tabular}{|c|c|c|c|c|c|c|}
\hline Data & $\begin{array}{c}\text { Number of } \\
\text { upregulated DEGs }\end{array}$ & $\begin{array}{c}\text { Number of } \\
\text { downregulated DEGs }\end{array}$ & $\begin{array}{l}\text { Number of total } \\
\text { DEGs }\end{array}$ & $|\log 2 \mathrm{FC}|$ & $\begin{array}{c}p \\
\text { value }\end{array}$ & $\begin{array}{l}\text { Expression scale (if the data } \\
\text { normalization was performed) }\end{array}$ \\
\hline GSE23586 & 7 & 69 & 76 & $>1$ & $<0.05$ & True \\
\hline GSE16134 & 188 & 48 & 236 & $>1$ & $<0.05$ & False \\
\hline GSE10334 & 152 & 42 & 194 & $>1$ & $<0.05$ & False \\
\hline GSE79705 & 219 & 291 & 510 & $>0$ & $<0.05$ & True \\
\hline
\end{tabular}

(CV) [31]. A SVM classifier model was established by using data from GSE16134 as the training set and test set, where the samples of the GSE16134 dataset were split into 60\%: $40 \%$ for the training set and test set, respectively. Data from the other three datasets (GSE23586, GSE10334, and GSE79705) were used as the validation set. The decision function method was used to obtain the score for each sample. Next, receiver operating characteristic (ROC) curves for the four datasets were generated by using the pROC package and displayed using the ggplot2 package in $\mathrm{R}$.

2.9. Targeting Relationships between Transcription Factors (TFs) and Crosstalk Genes. Transcription factor- (TF-) target gene regulation pairs were obtained and downloaded from TRRUST [32], cGRNB [33], HTRIdb [34], ORTI [35], and TRANSFAC [36] databases. The TF-target gene interaction pairs corresponding to the feature selection-obtained crosstalk genes were extracted and used for constructing a TFtarget gene interaction network with visualization using Cytoscape software [37].

2.10. Pathway Analysis of the Crosstalk Genes. Human data describing relationships between signaling pathways and genes were downloaded from the KEGG database, and all pathways related to the feature selection-obtained crosstalk genes were extracted. The expression levels of these crosstalk gene-related pathways were plotted as a heatmap, and differential expression analysis was performed and applied to the four periodontitis-related datasets to identify the DEPs (differentially expressed pathways) using the $\mathrm{R}$ package limma. For the three datasets (GSE23586, GSE16134, and GSE79705), the pathways with $p$ value $<0.05$ and $|\operatorname{logFC}| \geq$ 1 were regarded as differentially expressed pathways (DEPs), while for the dataset GSE79705, the pathways with $p$ value < 0.05 and $|\log \mathrm{FC}|>0$ were regarded as DEPs.

2.11. Classification Performance of the Core Crosstalk Genes. The genes related to periodontitis were downloaded from the DisGeNET database [26]. The overlap between the periodontitis-related genes obtained from the DisGeNET database and the feature selection-obtained crosstalk genes was analyzed, and the overlapping genes were termed core crosstalk genes. The corresponding expression values of these overlapping genes in the four periodontitis-related datasets were obtained, and ROC curves were drawn.

\section{Results}

3.1. Included Periodontitis-Related Datasets. Four datasets pertaining to periodontitis (i.e., GSE23586, GSE16134, GSE10334, and GSE79705) were included and analyzed. Table 1 provides key details regarding the included datasets.

3.2. Identification of Periodontitis-Related DEGs and Their Functions. Table 2 shows the number of up- and downregulated DEGs that were identified in each of the four datasets. 


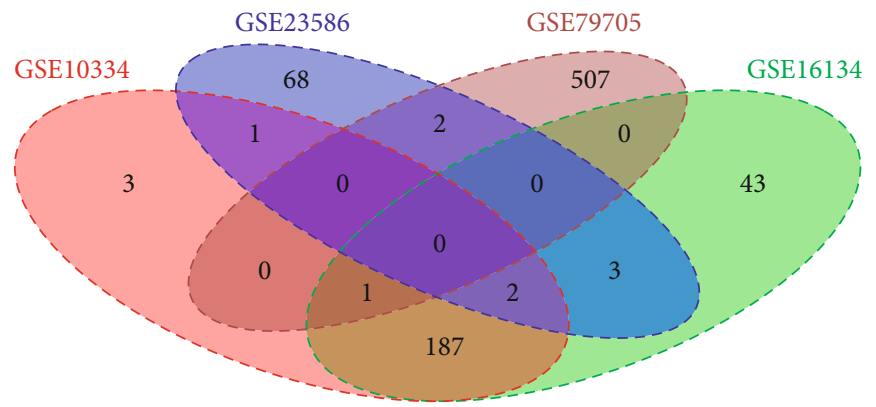

FIGURE 2: Venn diagram showing the overlap between DEGs identified in the four periodontitis-related datasets.
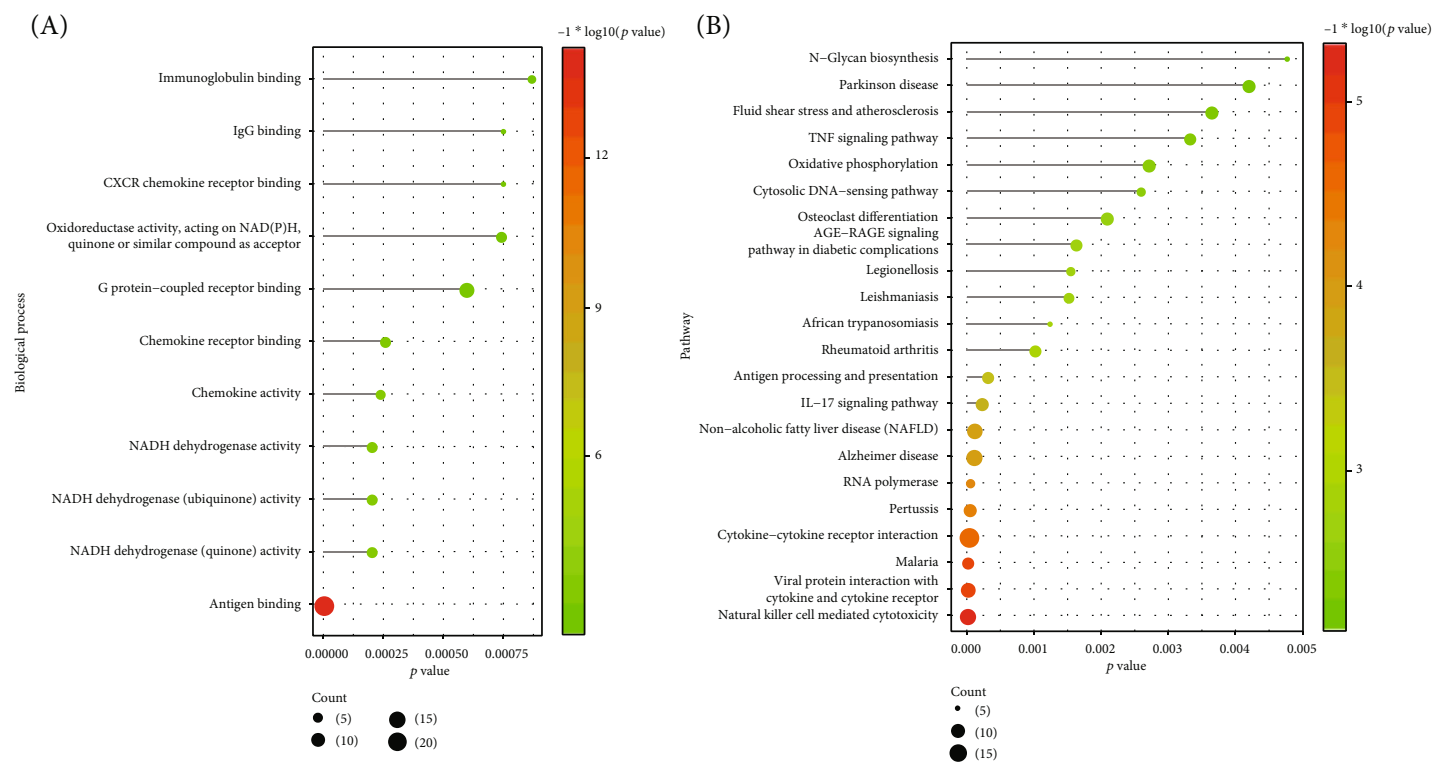

(a)

(A)

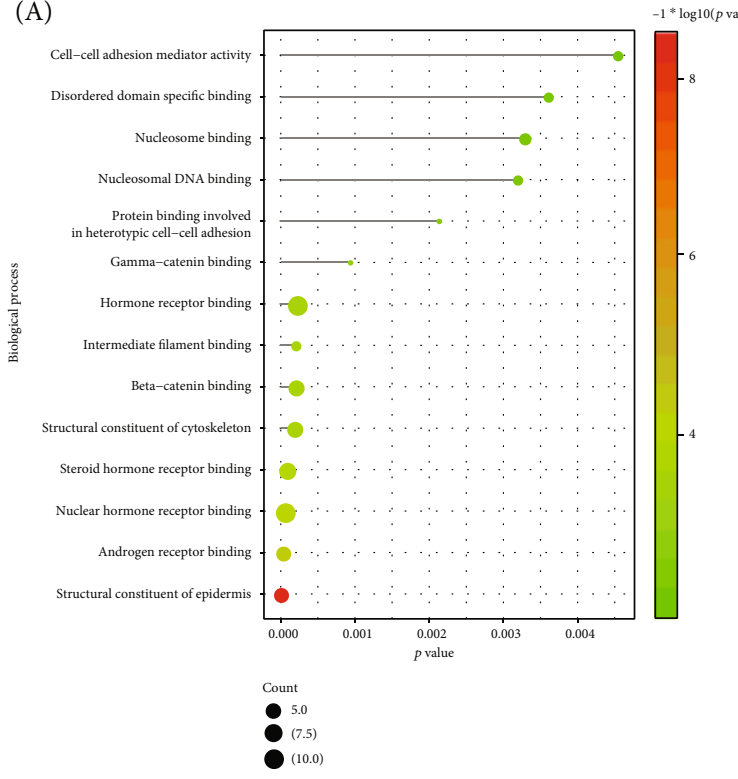

(B)

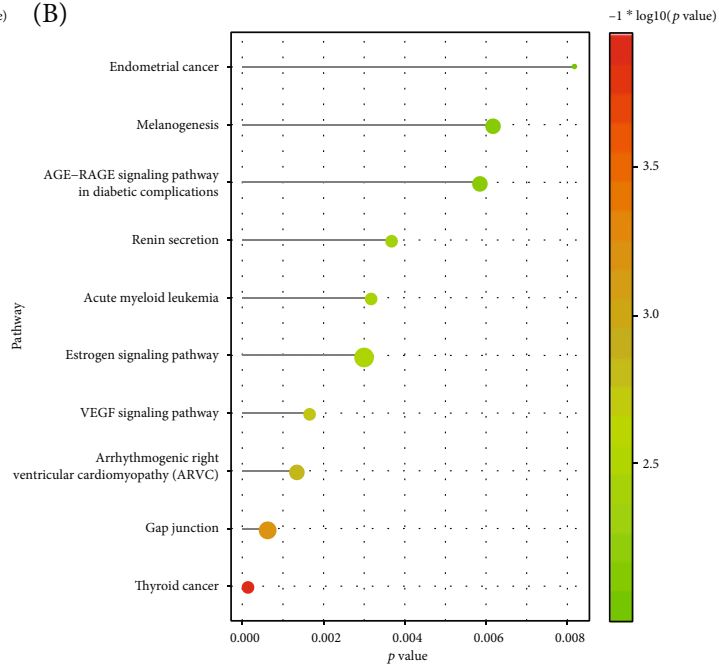

Count

(6)

(b)

FIGURE 3: Functional enrichment analysis of up- and downregulated periodontitis-related DEGs. (a) The functional terms (the biological processes (A) and KEGG pathways (B)) enriched by upregulated periodontitis-related DEGs. (b) The functional terms (the biological processes (A) and KEGG pathways (B)) enriched by downregulated periodontitis-related DEGs. 


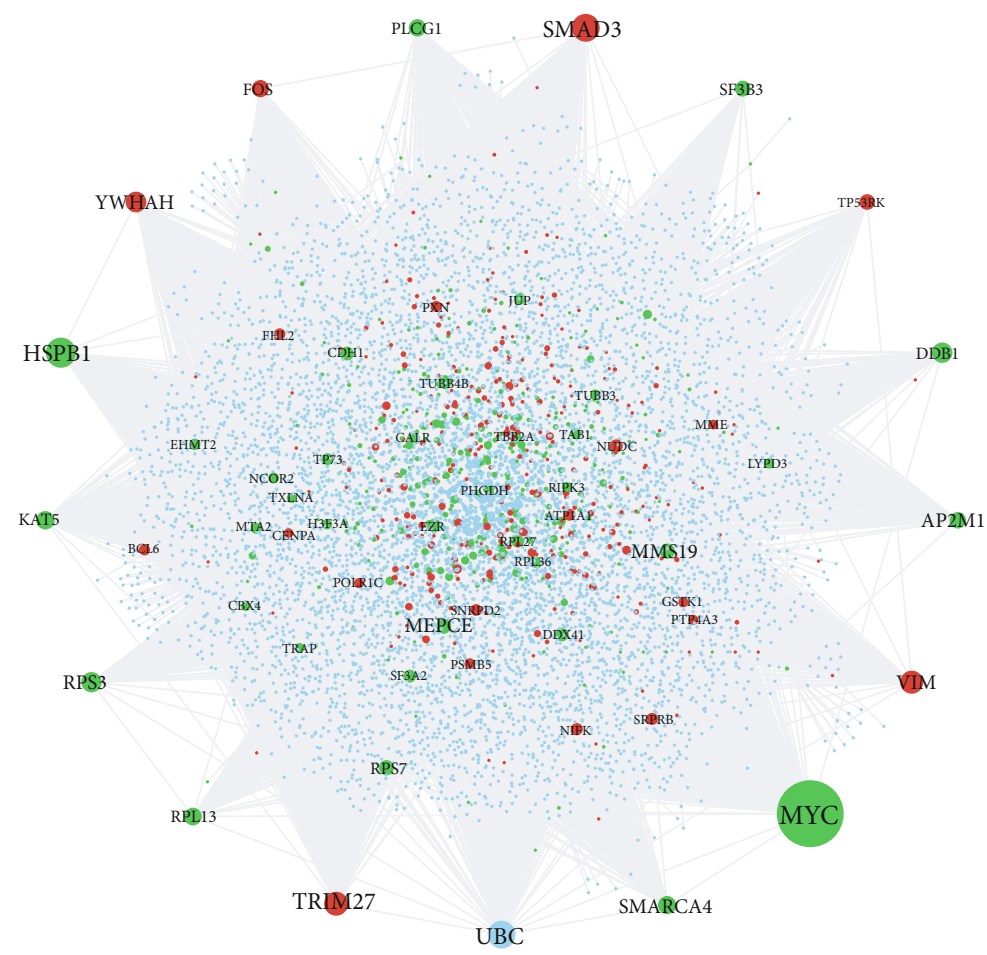

DEG_up
DEG_down
Other_gene

FIGURE 4: Protein-protein interaction (PPI) network associated with periodontitis. Red nodes represent the DEGs which were upregulated in periodontitis, green nodes represent the DEGs which were downregulated in periodontitis, and blue nodes represent other genes which were not DEGs but interacted with DEGs.

Figure 2 depicts a Venn diagram showing the overlap of DEGs identified from the four datasets. A total of 816 DEGs including 405 upregulated genes and 411 downregulated genes were used for the following analyses. Figure 3 shows the biological processes and signaling pathways in which the upand downregulated DEGs were significantly enriched.

3.3. The Hub Genes Identified by the Periodontitis-Related PPI Network. Figure 4 depicts a PPI network based on the periodontitis-related DEGs, and Table 3 shows the topological characteristics of the top 30 nodes in the PPI network. As seen in Table 3, several genes with the highest degree were identified as hub genes. These upregulated DEGs included SMAD3, TRIM27, VIM, YWHAH, and FOS, and downregulated DEGs included MYC, HSPB1, DDB1, RPS3, KAT5, SMARCA4, RPL13, and PLCG1.

3.4. Crosstalk Genes Bridging Alzheimer's Disease and Periodontitis. In total, 51 upregulated crosstalk genes and 41 downregulated crosstalk genes were identified and are listed in Table 4. The crosstalk gene-related PPI network as shown in Figure 5 consisted of 3496 nodes and 5141 edges. The topological characteristics of the top 30 nodes in this network are presented in Table 5 . Hub crosstalk genes with the highest degree included MYC, HSPB1, VIM, KAT5, RPL13, FOS, and CDH1.
3.5. Crosstalk Genes Obtained by Feature Selection. A total of 48 crosstalk genes were selected by using the Boruta algorithm (Figure 6(a)). In addition, 62 crosstalk genes were selected by using the RFE algorithm (Figure 6(b)). All 48 genes obtained by using the Boruta algorithm were included in the 62 genes obtained by the RFE algorithm, indicating that this 48-gene set was representative of the characteristics of all 92 crosstalk genes.

3.6. Classification Accuracy Using the Feature SelectionObtained Crosstalk Genes. The 48 genes obtained by SVM feature selection were not shown in all of the four periodontitis-related datasets. The gene expression profiles of GSE16134 and GSE10334 included all of the 48 crosstalk genes, whereas GSE23586 included 44 crosstalk genes and GSE79705 included 45 crosstalk genes. The classification performance of these 48 crosstalk genes for the four datasets is shown in Table 6. For the test set GSE16134 and the validation set GSE10334, the accuracy performance was high at 91.94\% and $88.26 \%$, respectively. By comparison, the performance for the other two datasets GSE23586 and GSE79705 was low at $50 \%$ and $66.66 \%$, respectively.

3.7. ROC Curves for the Four Periodontitis-Related Datasets. As shown in Figure 7, the AUC (area under the curve) values for the GSE16134 test set and the GSE10334 validation set 
TABLE 3: Topological characteristics of the top 30 nodes in the periodontitis-related PPI network, ranked in descending order of degree.

\begin{tabular}{|c|c|c|c|c|c|c|c|}
\hline Node & Label & Degree & $\begin{array}{c}\text { Average shortest path } \\
\text { length }\end{array}$ & $\begin{array}{l}\text { Betweenness } \\
\text { centrality }\end{array}$ & $\begin{array}{l}\text { Closeness } \\
\text { centrality }\end{array}$ & $\begin{array}{l}\text { Clustering } \\
\text { coefficient }\end{array}$ & $\begin{array}{c}\text { Topological } \\
\text { coefficient }\end{array}$ \\
\hline MYC & DEG_down & 1027 & 2.432727 & 0.177217 & 0.411061 & 0.001896 & 0.002805 \\
\hline HSPB1 & DEG_down & 433 & 2.638061 & 0.059908 & 0.379066 & 0.00278 & 0.005213 \\
\hline SMAD3 & DEG_up & 396 & 2.667636 & 0.052167 & 0.374864 & 0.004283 & 0.005425 \\
\hline UBC & & 395 & 2.112 & 0.241673 & 0.473485 & 0.004524 & 0.007004 \\
\hline TRIM27 & DEG_up & 334 & 2.864727 & 0.046792 & 0.349073 & 0.000791 & 0.006306 \\
\hline VIM & DEG_up & 320 & 2.705212 & 0.037607 & 0.369657 & 0.005251 & 0.007619 \\
\hline YWHAH & DEG_up & 282 & 2.838788 & 0.034115 & 0.352263 & 0.001868 & 0.00865 \\
\hline DDB1 & DEG_down & 276 & 2.76497 & 0.029677 & 0.361668 & 0.003531 & 0.008012 \\
\hline RPS3 & DEG_down & 275 & 2.844848 & 0.015036 & 0.351513 & 0.019323 & 0.014779 \\
\hline KAT5 & DEG_down & 245 & 2.717697 & 0.028224 & 0.367959 & 0.00716 & 0.007732 \\
\hline SMARCA4 & DEG_down & 242 & 2.715758 & 0.021646 & 0.368221 & 0.010116 & 0.008636 \\
\hline RPL13 & DEG_down & 234 & 2.755152 & 0.015773 & 0.362956 & 0.024541 & 0.011238 \\
\hline PLCG1 & DEG_down & 227 & 2.885091 & 0.030011 & 0.34661 & 0.002144 & 0.009614 \\
\hline FOS & DEG_up & 226 & 2.880727 & 0.024538 & 0.347135 & 0.003265 & 0.009974 \\
\hline AP2M1 & DEG_down & 214 & 2.850182 & 0.025582 & 0.350855 & 0.007635 & 0.010848 \\
\hline SF3B3 & DEG_down & 203 & 2.765576 & 0.015188 & 0.361588 & 0.014583 & 0.010661 \\
\hline TP53RK & DEG_up & 200 & 2.871394 & 0.013972 & 0.348263 & 0.01608 & 0.012798 \\
\hline MMS19 & DEG_down & 195 & 2.806909 & 0.022335 & 0.356264 & 0.004705 & 0.008763 \\
\hline RPS7 & DEG_down & 193 & 2.88303 & 0.008126 & 0.346857 & 0.028335 & 0.016367 \\
\hline MEPCE & DEG_down & 189 & 2.905212 & 0.0145 & 0.344209 & 0.005066 & 0.012337 \\
\hline TUBB4B & DEG_down & 173 & 2.880364 & 0.008167 & 0.347178 & 0.007595 & 0.019412 \\
\hline $\mathrm{CDH} 1$ & DEG_down & 171 & 2.888485 & 0.016186 & 0.346202 & 0.005848 & 0.011775 \\
\hline EZR & DEG_down & 170 & 2.906667 & 0.016295 & 0.344037 & 0.004107 & 0.01554 \\
\hline DDX41 & DEG_down & 168 & 2.950061 & 0.009545 & 0.338976 & 0.007057 & 0.017509 \\
\hline NUDC & DEG_up & 160 & 2.943758 & 0.016886 & 0.339702 & 0.00283 & 0.013954 \\
\hline SF3A2 & DEG_down & 156 & 2.949818 & 0.009075 & 0.339004 & 0.009926 & 0.01593 \\
\hline RPL27 & DEG_down & 147 & 2.912727 & 0.003949 & 0.343321 & 0.043985 & 0.020057 \\
\hline NIFK & DEG_up & 145 & 3.107879 & 0.011111 & 0.321763 & 0.010345 & 0.016053 \\
\hline SRPRB & DEG_up & 143 & 2.945333 & 0.017545 & 0.33952 & 0.001674 & 0.015983 \\
\hline ATP1A1 & DEG_up & 140 & 2.816606 & 0.011269 & 0.355037 & 0.009147 & 0.013032 \\
\hline
\end{tabular}

were high at $95.77 \%$ and $90.53 \%$, respectively, congruent with the results in Table 6 . It was thus inferred that the classifier performance was adequate only when the sample sizes of the validation sets were similar to those of the training and test datasets, and therefore, poor performance was noted for GSE23586 and GSE79705 having much lower sample numbers.

3.8. The Identification of Transcription Factors Regulating the Crosstalk Genes. As shown in Figure 8, the TF-crosstalk gene target network consisted of 388 nodes and 1178 edges. Several transcription factors which were also DEGs played critical roles by regulating the most number of crosstalk genes, for example, FOS, MEF2C, and USF2 (Table 7).

3.9. Signaling Pathways Enriched in the Crosstalk Genes. From the 48 feature selection-obtained crosstalk genes, 37 crosstalk genes were found among gene-pathway interaction pair data in the KEGG database. 137 KEGG pathways corre- sponded to these 37 crosstalk genes. Figure 9 shows the expression values of these 137 pathways in the four periodontitis-related datasets.

The numbers of crosstalk gene-related DEPs obtained from each of the four periodontitis-related datasets are listed in Table 8. The interaction relationships between crosstalk genes and DEPs are depicted in Figure 10, showing that several DEPs were dysregulated in at least two datasets, including cytokine-related pathways (cytokine-cytokine receptor interaction, chemokine, and IL-17), immune cell-related pathways ( $\mathrm{T}$ cell receptor, B cell receptor, Th1 and Th2 cell differentiation, Th17 cell differentiation, natural killer cellmediated cytotoxicity, and osteoclast differentiation), JAKSTAT signaling, NOD-like receptor signaling, MAPK signaling, Toll-like receptor signaling, NF-kappa B signaling, and C-type lectin receptor signaling.

3.10. The Identification of Core Crosstalk Genes. Among the 48 feature selection-obtained crosstalk genes and 
TABLE 4: The 51 crosstalk genes that were upregulated and 41 crosstalk genes which were downregulated in periodontitis.

Regulation pattern in periodontitis

Crosstalk genes linking periodontitis and Alzheimer's disease

AMIGO1 (adhesion molecule with Ig-like domain 1; gene ID: 57463)

ARG2 (arginase 2; gene ID: 384)

BDKRB2 (bradykinin receptor B2; gene ID: 624)

BRI3 (brain protein I3; gene ID: 25798)

C1D (C1D nuclear receptor corepressor; gene ID: 10438)

C3 (complement C3; gene ID: 718)

C4A (complement C4A (Rodgers blood group); gene ID: 720)

C4B (complement C4B; gene ID: 721)

C4B_2 (complement component 4B (Chido blood group), copy 2; gene ID: 100293534) CASP7 (caspase 7; gene ID: 840)

CD177 (CD177 molecule; gene ID: 57126)

CD38 (CD38 molecule; gene ID: 952)

CHCHD10 (coiled-coil-helix-coiled-coil-helix domain containing 10; gene ID: 400916)

COX8A (cytochrome c oxidase subunit 8A; gene ID: 1351)

CSF3 (colony-stimulating factor 3; gene ID: 1440)

CTGF (cellular communication network factor 2; gene ID: 1490) CTSS (cathepsin S; gene ID: 1520)

CXCL1 (C-X-C motif chemokine ligand 1; gene ID: 2919)

CXCL12 (C-X-C motif chemokine ligand 12; gene ID: 6387)

CXCL8 (C-X-C motif chemokine ligand 8; gene ID: 3576)

CXCR4 (C-X-C motif chemokine receptor 4; gene ID: 7842)

ENPP2 (ectonucleotide pyrophosphatase/phosphodiesterase 2; gene ID: 5168)

FCGR3A (Fc fragment of IgG receptor IIIa; gene ID: 2214)

FCGR3B (Fc fragment of IgG receptor IIIb; gene ID: 2215)

FHL2 (four and a half LIM domains 2; gene ID: 2274)

Upregulated

FOS (Fos proto-oncogene, AP-1 transcription factor subunit; gene ID: 2353)

FOXO1 (forkhead box O1; gene ID: 2308)

GSTK1 (glutathione S-transferase kappa 1; gene ID: 373156)

GSTO1 (glutathione S-transferase omega 1; gene ID: 9446)

HCLS1 (hematopoietic cell-specific Lyn substrate 1; gene ID: 3059)

IGFBP7 (insulin-like growth factor binding protein 7; gene ID: 3490)

IL1B (interleukin-1 beta; gene ID: 3553)

LYZ (lysozyme; gene ID: 4069)

MAK16 (MAK16 homolog; gene ID: 84549)

MEF2C (myocyte-specific enhancer factor 2C; gene ID: 4208)

MME (membrane metalloendopeptidase; gene ID: 4311)

MMP1 (matrix metallopeptidase 1; gene ID: 4312)

MMP3 (matrix metallopeptidase 3; gene ID: 4314)

MS4A1 (membrane-spanning 4-domains A1; gene ID: 931)

MSRA (methionine sulfoxide reductase A; gene ID: 4482)

MZB1 (marginal zone B and B1 cell-specific protein; gene ID: 51237)

NDUFB8 (NADH:ubiquinone oxidoreductase subunit B8; gene ID: 4714)

PECAM1 (platelet endothelial cell adhesion molecule 1; gene ID: 5175)

PLAT (plasminogen activator, tissue type; gene ID: 5327)

PSENEN (presenilin enhancer, gamma-secretase subunit; gene ID: 55851)

SEL1L (SEL1L adaptor subunit of ERAD E3 ubiquitin ligase; gene ID: 6400)

SERPINI1 (serpin family I member 1; gene ID: 5274)

ST6GAL1 (ST6 beta-galactoside alpha-2,6-sialyltransferase 1; gene ID: 6480)

VCAN (versican; gene ID: 1462)

VIM (vimentin; gene ID: 7431)

WARS2 (tryptophanyl tRNA synthetase 2, mitochondrial; gene ID: 10352)

ABCA12 (ATP binding cassette subfamily A member 12; gene ID: 26154)

ACE (angiotensin I converting enzyme; gene ID: 1636)

ADRB1 (adrenoceptor beta 1; gene ID: 153)

BCL2L2 (BCL2-like 2; gene ID: 599)

BRSK1 (BR serine/threonine kinase 1; gene ID: 84446)

CALML5 (calmodulin-like 5; gene ID: 51806)

CALR (calreticulin; gene ID: 811)

CD36 (CD36 molecule; gene ID: 948)

CDH1 (cadherin 1; gene ID: 999)

CDK5R1 (cyclin-dependent kinase 5 regulatory subunit 1; gene ID: 8851)

CTDSP2 (CTD small phosphatase 2; gene ID: 10106) 
TABLE 4: Continued.

Regulation pattern in periodontitis

Crosstalk genes linking periodontitis and Alzheimer's disease

DNM1 (dynamin 1; gene ID: 1759)

DSG1 (desmoglein 1; gene ID: 1828)

FLG (filaggrin; gene ID: 2312)

GJA1 (gap junction protein alpha 1; gene ID: 2697)

GRHL3 (grainyhead-like transcription factor 3; gene ID: 57822)

GRIN3B (glutamate ionotropic receptor NMDA type subunit 3B; gene ID: 116444)

HM13 (histocompatibility minor 13; gene ID: 81502)

HMGCR (3-hydroxy-3-methylglutaryl-CoA reductase; gene ID: 3156)

HSPB1 (heat shock protein family B (small) member 1; gene ID: 3315)

KAT5 (lysine acetyltransferase 5; gene ID: 10524)

MAP3K12 (mitogen-activated protein kinase kinase kinase 12; gene ID: 7786)

MED12 (mediator complex subunit 12; gene ID: 9968)

MFN2 (mitofusin 2; gene ID: 9927)

MYC (MYC proto-oncogene, bHLH transcription factor; gene ID: 4609)

NEFL (neurofilament light; gene ID: 4747)

NES (exportin 1; gene ID: 7514)

NGF (nerve growth factor; gene ID: 4803)

NOS3 (nitric oxide synthase 3; gene ID: 4846)

NPTXR (neuronal pentraxin receptor; gene ID: 23467)

PLXNA3 (plexin A3; gene ID: 55558)

PPIL2 (peptidylprolyl isomerase-like 2; gene ID: 23759)

PTGS1 (prostaglandin-endoperoxide synthase 1; gene ID: 5742)

RPL13 (ribosomal protein L13; gene ID: 6137)

SCIMP (SLP adaptor and CSK interacting membrane protein; gene ID: 388325)

SPPL2B (signal peptide peptidase-like 2B; gene ID: 56928)

TIAF1 (TGFB1-induced antiapoptotic factor 1; gene ID: 9220)

TP73 (tumor protein P73; gene ID: 7161)

TYRP1 (tyrosinase-related protein 1; gene ID: 7306)

USF2 (upstream transcription factor 2, C-Fos interacting; gene ID: 7392)

VEGFA (vascular endothelial growth factor A; gene ID: 7422)

periodontitis-related genes in the DisGeNET database, 12 common genes were identified as core crosstalk genes including C3, C4A, C4B, CXCL12, FCGR3A, FCGR3B, HSPB1, IL1B, MME, MMP3, PLAT, and VEGFA (Table 9). Among these 12 genes, 6 genes, C4A, C4B, CXCL12, FCGR3A, IL1B, and MMP3, were found associated with chronic periodontitis.

Figure 11 shows ROC curves for the 6 chronic periodontitis-related genes for each of the four datasets. An AUC value of more than $80 \%$ was presented by three genes, C4A, C4B, and CXCL12, for the dataset GSE10334, 5 genes, C4A, C4B, CXCL12, FCGR3A, and IL1B, for GSE16134, 3 genes, CXCL12, IL1B, and MMP3, for GSE23586, and all 6 genes for GSE79705. The 3 genes, C4A, C4B, and CXCL1, had the highest classification accuracy when the datasets with small samples (GSE23586 and GSE79705) were not considered.

\section{Discussion}

The present study addressed shared genetic mechanisms and molecular links between periodontitis and Alzheimer's diseases by identifying gene expression, signaling pathways, and TFs that were most robustly associated with both these diseases. These findings are largely substantiated by preexisting experimental data.

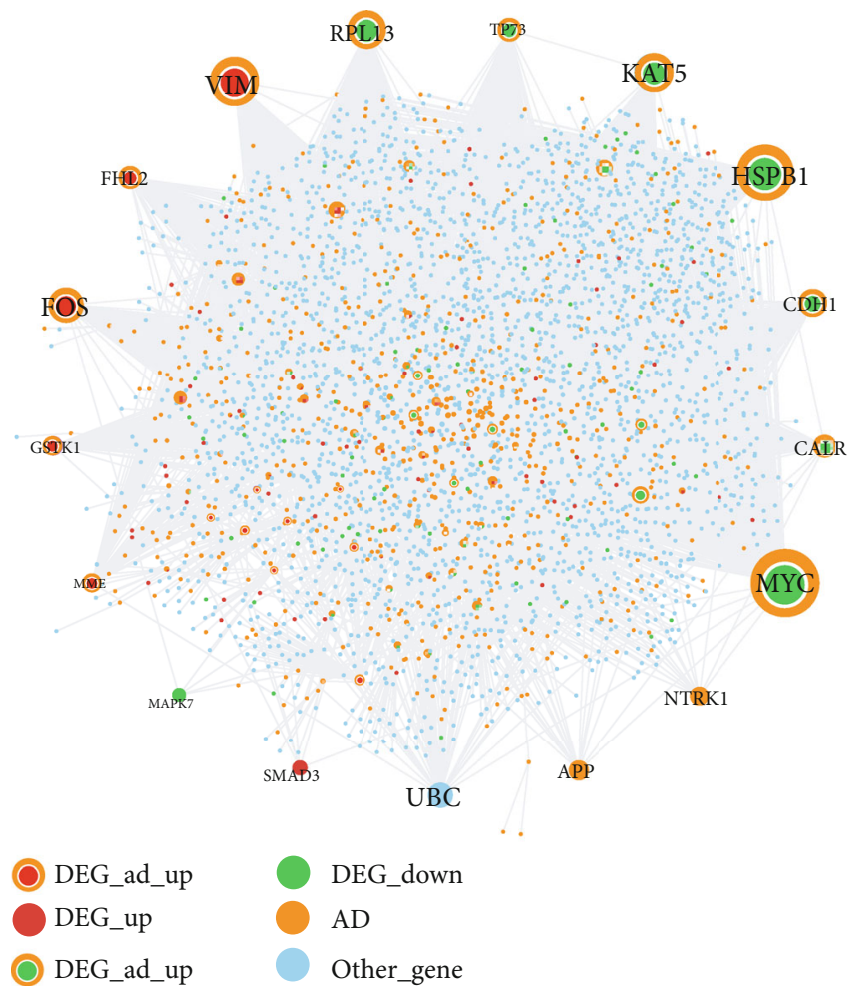

FIgURE 5: The crosstalk gene-related PPI network. 
TABLE 5: Topological characteristics of the top 30 nodes in the crosstalk gene-related PPI network, ranked in descending order of degree.

\begin{tabular}{|c|c|c|c|c|c|c|c|}
\hline Node & Label & Degree & $\begin{array}{l}\text { Average shortest path } \\
\text { length }\end{array}$ & $\begin{array}{l}\text { Betweenness } \\
\text { centrality }\end{array}$ & $\begin{array}{l}\text { Closeness } \\
\text { centrality }\end{array}$ & $\begin{array}{l}\text { Clustering } \\
\text { coefficient }\end{array}$ & $\begin{array}{c}\text { Topological } \\
\text { coefficient }\end{array}$ \\
\hline MYC & DEG_AD_down & 1027 & 2.127525 & 0.466698 & 0.47003 & $5.03 E-04$ & 0.001842 \\
\hline HSPB1 & DEG_AD_down & 433 & 2.36959 & 0.184598 & 0.422014 & 0.001636 & 0.003351 \\
\hline VIM & DEG_AD_up & 320 & 2.663301 & 0.116611 & 0.375474 & 0.001763 & 0.005783 \\
\hline KAT5 & DEG_AD_down & 245 & 2.566359 & 0.089476 & 0.389657 & 0.003011 & 0.005621 \\
\hline RPL13 & DEG_AD_down & 234 & 2.589729 & 0.089708 & 0.386141 & 0.001394 & 0.005672 \\
\hline FOS & DEG_AD_up & 226 & 2.883151 & 0.083642 & 0.346843 & $1.97 E-04$ & 0.017606 \\
\hline $\mathrm{CDH} 1$ & DEG_AD_down & 171 & 2.93191 & 0.058428 & 0.341075 & 0 & 0.025911 \\
\hline TP73 & DEG_AD_down & 137 & 2.61483 & 0.046781 & 0.382434 & 0.006333 & 0.009173 \\
\hline FHL2 & DEG_AD_up & 136 & 2.641085 & 0.057192 & 0.378632 & 0.004031 & 0.00889 \\
\hline CALR & DEG_AD_down & 135 & 2.812464 & 0.046142 & 0.35556 & 0.002764 & 0.010949 \\
\hline GSTK1 & DEG_AD_up & 111 & 2.988459 & 0.035152 & 0.334621 & 0 & 0.026289 \\
\hline MME & DEG_AD_up & 104 & 2.836988 & 0.040386 & 0.352487 & 0.001307 & 0.011694 \\
\hline MED12 & DEG_AD_down & 91 & 2.695615 & 0.030682 & 0.370973 & 0.006105 & 0.012517 \\
\hline CASP7 & DEG_AD_up & 90 & 2.901039 & 0.027131 & 0.344704 & 0.005243 & 0.015603 \\
\hline DNM1 & DEG_AD_down & 84 & 2.960185 & 0.029086 & 0.337817 & 0.001147 & 0.023716 \\
\hline FOXO1 & DEG_AD_up & 67 & 2.977784 & 0.017403 & 0.33582 & 0.004071 & 0.026586 \\
\hline CXCR4 & DEG_AD_up & 65 & 2.998557 & 0.020851 & 0.333494 & 0.003846 & 0.028808 \\
\hline NOS3 & DEG_AD_down & 62 & 2.963647 & 0.018786 & 0.337422 & 0.002644 & 0.027957 \\
\hline NDUFB8 & DEG_AD_up & 56 & 3.042412 & 0.02136 & 0.328687 & 0 & 0.027447 \\
\hline UBC & Other gene & 53 & 2.084824 & 0.149265 & 0.479657 & 0.015965 & 0.027336 \\
\hline NES & DEG_AD_down & 53 & 2.920946 & 0.017012 & 0.342355 & 0.007983 & 0.022954 \\
\hline CDK5R1 & DEG_AD_down & 50 & 3.0176 & 0.0133 & 0.331389 & 0.002449 & 0.037222 \\
\hline NEFL & DEG_AD_down & 49 & 2.913445 & 0.014886 & 0.343236 & 0.008503 & 0.024673 \\
\hline C3 & DEG_AD_up & 43 & 3.043278 & 0.01705 & 0.328593 & 0 & 0.042101 \\
\hline SEL1L & DEG_AD_up & 42 & 3.036065 & 0.013066 & 0.329374 & 0 & 0.045635 \\
\hline DSG1 & DEG_AD_down & 41 & 3.046163 & 0.00857 & 0.328282 & 0 & 0.068464 \\
\hline GJA1 & DEG_AD_down & 41 & 3.035199 & 0.015703 & 0.329468 & 0 & 0.04037 \\
\hline MEF2C & DEG_AD_up & 41 & 3.039238 & 0.008676 & 0.32903 & 0 & 0.068892 \\
\hline LYZ & DEG_AD_up & 40 & 3.042123 & 0.008578 & 0.328718 & 0 & 0.065086 \\
\hline GSTO1 & DEG_AD_up & 38 & 2.820254 & 0.010905 & 0.354578 & 0.019915 & 0.030356 \\
\hline
\end{tabular}

Six genes C4A, C4B, CXCL12, FCGR3A, IL1B, and MMP3 were identified as the most significant crosstalk genes linking chronic periodontitis and Alzheimer's disease. C4B and $\mathrm{C} 4 \mathrm{~A}$, respectively, encode the basic and acidic forms of the complement factor 4 , and $\mathrm{C} 4$ gene deficiency has been noted to predispose the development of severe chronic periodontitis [38]. In $\mathrm{AD}$, the expression levels of C4 mRNA were shown to be 3.27-fold increased in temporal cortex samples as compared to controls [39]. The CXCL12 (C-X-C motif chemokine ligand 12) gene is the ligand of the C-X-C motif chemokine receptor 4 (CXCR4). CXCL12 expression in the gingival crevicular fluid of periodontitis patients was shown to be significantly higher than that of healthy subjects, suggesting that it might play a role in enhancing neutrophil migration and further the progression of periodontitis [40]. A decreased level of CXCL12 in Alzheimer's disease has been documented as affecting cognitive function, impairing learning and memory [41]. FCGR3A (Fc fragment of IgG receptor IIIa) encodes a receptor for the Fc portion of immunoglobu- lin G, and FCGR3A polymorphisms are shown to confer susceptibility to periodontitis in Caucasians [42]. In AD, the Fc gamma receptor $(\mathrm{Fc} \gamma \mathrm{R})$ was recently found to exacerbate neurodegeneration [43]. Cytokines are considered a primary link between chronic periodontitis and Alzheimer's disease as they can enter systemic circulation through periodontal pockets [6]. The classical proinflammatory cytokine, IL1B, is elevated in periodontitis and can induce resorption of alveolar bone [44]. In AD, IL1B gene polymorphisms are linked to disease susceptibility [45]. MMP3 (matrix metalloproteinase 3) is implicated in the progression of chronic periodontitis and can degrade the periodontal tissue matrix [46]. Elevated brain levels of MMP3 have been associated with the duration of Alzheimer's disease, and it has been found to increase the activity of MMP9, thereby indirectly promoting aggregation and cerebral accumulation of tau deposits [47].

More interestingly, the six genes discussed in the last paragraph were also found to be the molecular crosstalks in 


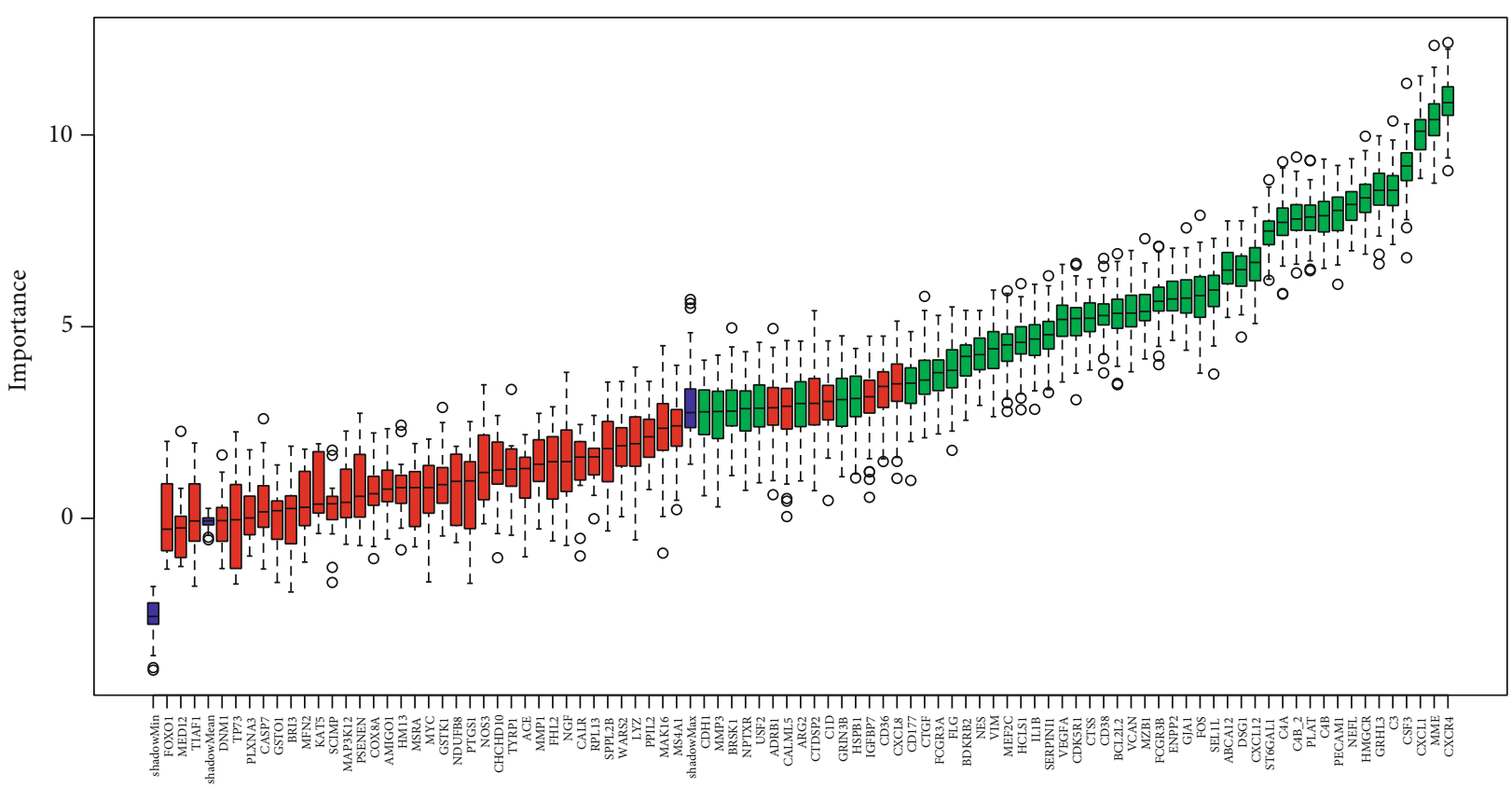

(a) Boruta algorithm to obtain genes

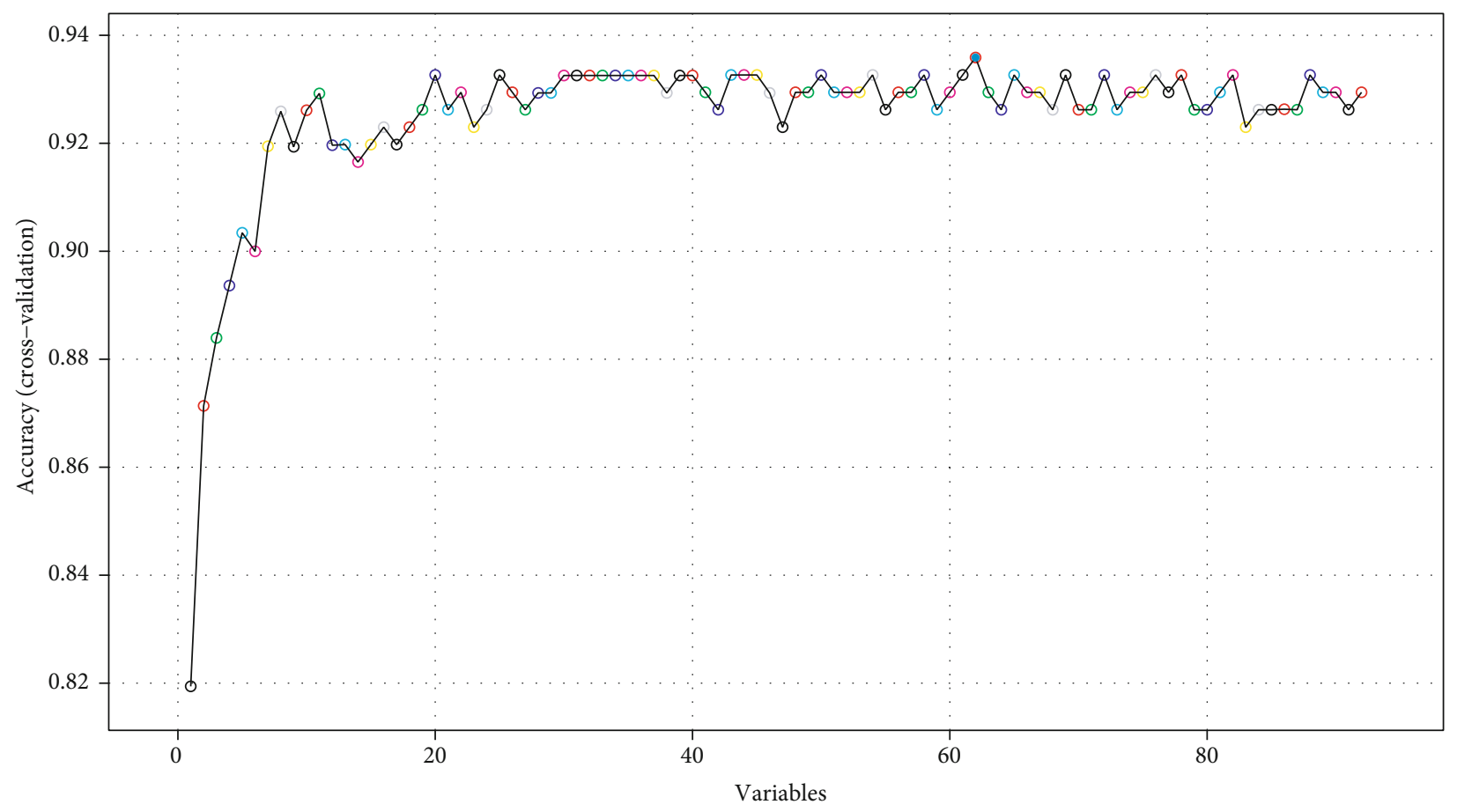

(b) RFE algorithm to obtain genes

FIGURE 6: 48 crosstalk genes selected by using the Boruta algorithm (a) and 62 crosstalk genes selected by using the RFE algorithm (b).

linking the peripheral immune system and central nervous system (CNS). It has been well demonstrated that the periodontal disease-evoked peripheral systemic host immune response can aggravate the progression of neuroinflammation and neurodegeneration in Alzheimer's disease by switching the microglia from the primed phenotype to an aggressive proinflammatory phenotype [48, 49]. The immune-inflammatory mediators (e.g., cytokines and chemokines) abundantly expressed during periodontal inflam- mation can circulate into the bloodstream and travel into the brain by crossing the blood-brain barrier (BBB) and impact the function of CNS [50]. Therefore, the crosstalk between the peripheral immune system and the CNS might be an important mechanism underlying periodontitis, increasing the risk of $\mathrm{AD}$. This paragraph will provide a description regarding the potential role of the six crosstalk genes in linking periodontal disease and $\mathrm{AD}$, especially by means of neuroimmune interaction. For example, the 


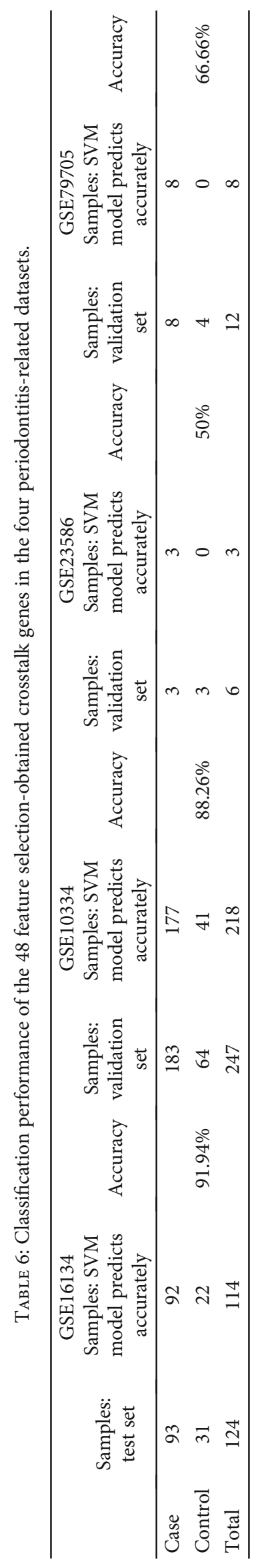




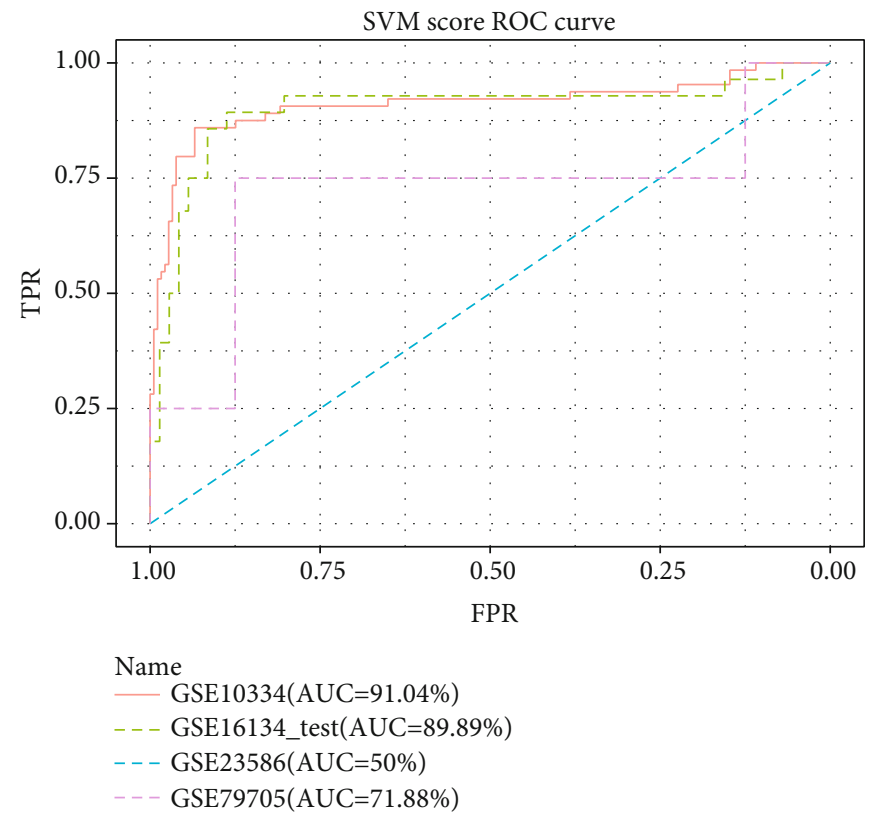

FIgURE 7: The ROC curves of four periodontitis-related datasets.

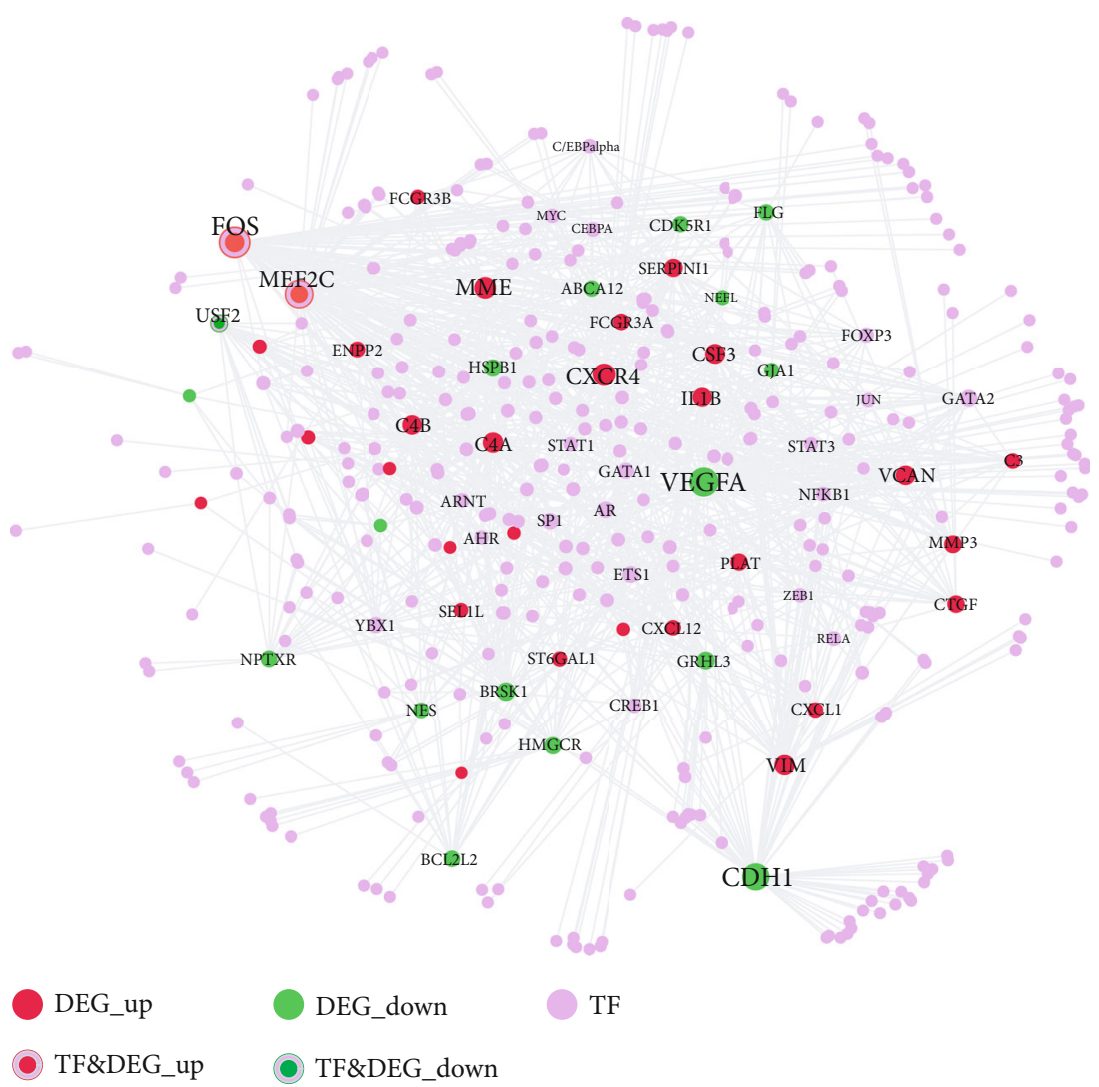

FiguRE 8: The transcription factor-crosstalk gene target network.

complement components $\mathrm{C} 4 \mathrm{~A}$ and $\mathrm{C} 4 \mathrm{~B}$ highly expressed in periodontal disease were found to modulate $\mathrm{T}$ cell immune response by stimulating the activation and migration of $\mathrm{T}$ cells [51-53]. The migration of $\mathrm{T}$ cells enhanced by $\mathrm{C} 4 \mathrm{~A}$ and $\mathrm{C} 4 \mathrm{~B}$ might allow $\mathrm{T}$ cells to traffic across the $\mathrm{BBB}$ and enter the brain. For another example, the chemokine and its receptor-composed system CXCL12/CXCR4-7 system were found to be a significant player of the neuroimmune interface [54]. On the one hand, the chemokine CXCL12 mediated the immune-inflammatory response in CNS by 
TABLE 7: Topological characteristics of the top 30 nodes in the TF-crosstalk gene target network, ranking in descending order.

\begin{tabular}{|c|c|c|c|c|c|c|c|}
\hline Node & Label & Degree & $\begin{array}{c}\text { Average shortest path } \\
\text { length }\end{array}$ & $\begin{array}{l}\text { Betweenness } \\
\text { centrality }\end{array}$ & $\begin{array}{l}\text { Closeness } \\
\text { centrality }\end{array}$ & $\begin{array}{l}\text { Clustering } \\
\text { coefficient }\end{array}$ & $\begin{array}{c}\text { Topological } \\
\text { coefficient }\end{array}$ \\
\hline FOS & DEG_up\&TF & 89 & 2.178295 & 0.206101 & 0.459075 & 0.020429 & 0.03825 \\
\hline VEGFA & DEG_down & 77 & 2.211886 & 0.197219 & 0.452103 & 0.010936 & 0.038456 \\
\hline MEF2C & DEG_up\&TF & 76 & 2.328165 & 0.159945 & 0.429523 & 0.004211 & 0.044258 \\
\hline $\mathrm{CDH} 1$ & DEG_down & 69 & 2.540052 & 0.159392 & 0.393693 & 0 & 0.086634 \\
\hline CXCR4 & DEG_up & 47 & 2.596899 & 0.075105 & 0.385075 & $9.25 E-04$ & 0.083836 \\
\hline MME & DEG_up & 44 & 2.664083 & 0.04365 & 0.375364 & 0 & 0.131313 \\
\hline $\mathrm{C} 4 \mathrm{~A}$ & DEG_up & 38 & 2.661499 & 0.029742 & 0.375728 & 0.01138 & 0.118617 \\
\hline VIM & DEG_up & 38 & 2.521964 & 0.060293 & 0.396516 & 0.012802 & 0.065789 \\
\hline CSF3 & DEG_up & 36 & 2.550388 & 0.031734 & 0.392097 & 0.019048 & 0.075137 \\
\hline $\mathrm{C} 4 \mathrm{~B}$ & DEG_up & 34 & 2.679587 & 0.018887 & 0.373192 & 0.012478 & 0.122037 \\
\hline VCAN & DEG_up & 34 & 2.726098 & 0.030567 & 0.366825 & 0 & 0.143717 \\
\hline IL1B & DEG_up & 33 & 2.741602 & 0.05746 & 0.36475 & 0 & 0.134602 \\
\hline BRSK1 & DEG_down & 30 & 2.741602 & 0.030404 & 0.36475 & 0 & 0.153788 \\
\hline SERPINI1 & DEG_up & 29 & 2.793282 & 0.019014 & 0.358002 & 0 & 0.127586 \\
\hline USF2 & $\begin{array}{c}\mathrm{DEG}_{-} \\
\text {down\&TF }\end{array}$ & 27 & 2.591731 & 0.027575 & 0.385842 & 0.045584 & 0.081607 \\
\hline GRHL3 & DEG_down & 27 & 2.775194 & 0.027067 & 0.360335 & 0 & 0.150732 \\
\hline HSPB1 & DEG_down & 26 & 2.780362 & 0.017981 & 0.359665 & 0 & 0.155678 \\
\hline MMP3 & DEG_up & 26 & 2.607235 & 0.024377 & 0.383548 & 0.036923 & 0.074984 \\
\hline CTGF & DEG_up & 25 & 2.788114 & 0.020669 & 0.358665 & 0 & 0.161905 \\
\hline HMGCR & DEG_down & 25 & 2.767442 & 0.041119 & 0.361345 & 0 & 0.16 \\
\hline GATA2 & $\mathrm{TF}$ & 24 & 2.095607 & 0.052687 & 0.477189 & 0.018116 & 0.104678 \\
\hline PLAT & DEG_up & 24 & 2.762274 & 0.028075 & 0.362021 & 0 & 0.173148 \\
\hline ETS1 & $\mathrm{TF}$ & 22 & 2.273902 & 0.03767 & 0.439773 & 0.012987 & 0.092022 \\
\hline NPTXR & DEG_down & 22 & 2.826873 & 0.020478 & 0.353748 & 0 & 0.154545 \\
\hline $\mathrm{AR}$ & $\mathrm{TF}$ & 21 & 2.24031 & 0.040698 & 0.446367 & 0.02381 & 0.105465 \\
\hline YBX1 & $\mathrm{TF}$ & 21 & 2.315245 & 0.037801 & 0.43192 & 0.004762 & 0.098928 \\
\hline BCL2L2 & DEG_down & 21 & 2.813953 & 0.018179 & 0.355372 & 0 & 0.144048 \\
\hline FCGR3A & DEG_up & 21 & 2.81137 & 0.020521 & 0.355699 & 0 & 0.192799 \\
\hline ABCA12 & DEG_down & 20 & 2.79845 & 0.010961 & 0.357341 & 0 & 0.2 \\
\hline SP1 & $\mathrm{TF}$ & 20 & 2.191214 & 0.037362 & 0.456368 & 0.026316 & 0.109167 \\
\hline CDK5R1 & DEG_down & 20 & 2.824289 & 0.017878 & 0.354071 & 0 & 0.156098 \\
\hline FLG & DEG_down & 20 & 2.653747 & 0.012281 & 0.376826 & 0.042105 & 0.093043 \\
\hline
\end{tabular}

recruiting lymphocytes and macrophages [55]. On the other hand, CXCL12 can lead to neurotoxicity and neurodegenerescence by activating the neuronal survival-associated $\mathrm{G}$ protein-activated inward rectifier $\mathrm{K}(+)$ (GIRK) [54]. FCGR3A (also named CD16) is essential for the antibodydependent cellular cytotoxicity (ADCC) mediated by natural killer (NK) cells [56]. The increased cytotoxic activity of NK cells was found to cause the dysregulation of protein kinase $\mathrm{C}$ and further led to the cognitive deficits in Alzheimer's disease, indicating the contribution of immunological factors to the dysfunction of CNS [57]. IL1B, which was upregulated in periodontitis and transported through the vascular circulation into the brain, was found to play promoting roles in neuroinflammation by enhancing the expression of leukocyte chemotactic chemokines, cell surface adhesion molecules, cyclooxygenases, and MMPs within the brain parenchyma
[58]. Likewise, MMP3 abundantly produced in periodontitis was also found to be associated with neuroinflammation via activating microglial cells, as well as participating in the BBB breakdown through the proteolysis of fibronectin and type IV collagen [59]. Taken together, the six crosstalk genes identified in the present research were well evidenced to be involved in periodontitis-triggered peripheral systemic host immune response caused CNS dysfunction in Alzheimer's disease.

Three transcription factors, FOS, MEF2C, and USF2, were identified as related to the regulation of the crosstalk genes and were also found to be dysregulated in chronic periodontitis. The proto-oncogene FOS (also named C-Fos) was found to be involved in the transcriptional regulation of collagenase and cell proliferation genes in periodontal gingival fibroblasts [60]. In $\mathrm{AD}, \mathrm{FOS}$ is reported to initiate amyloid- 


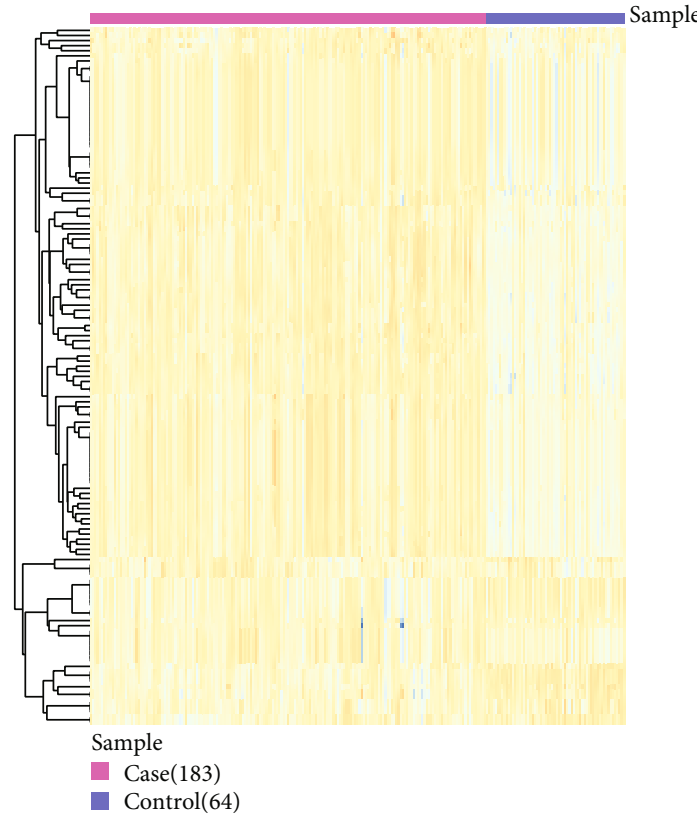

(a) GSE10334

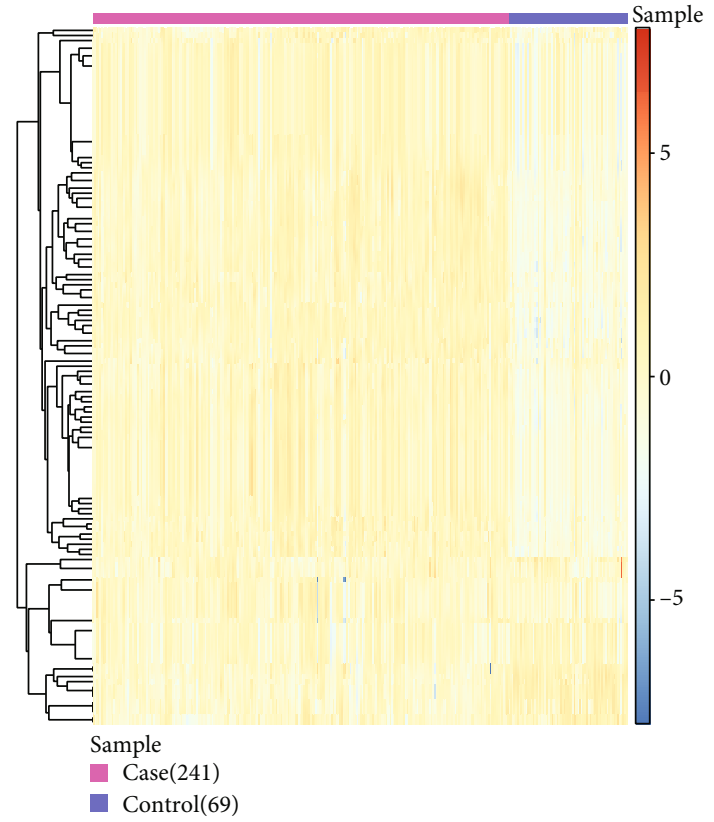

(b) GSE16134

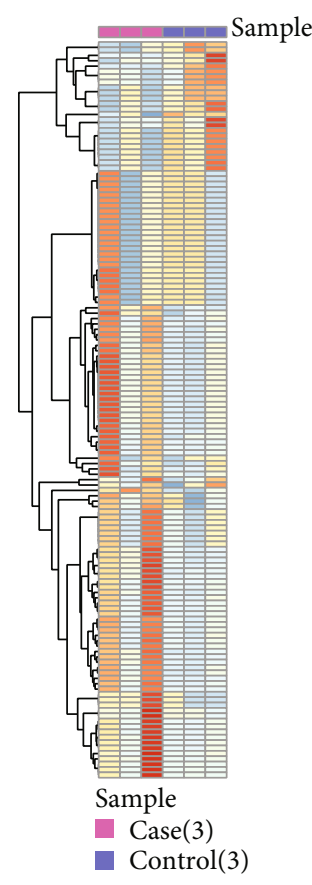

(c) GSE23586

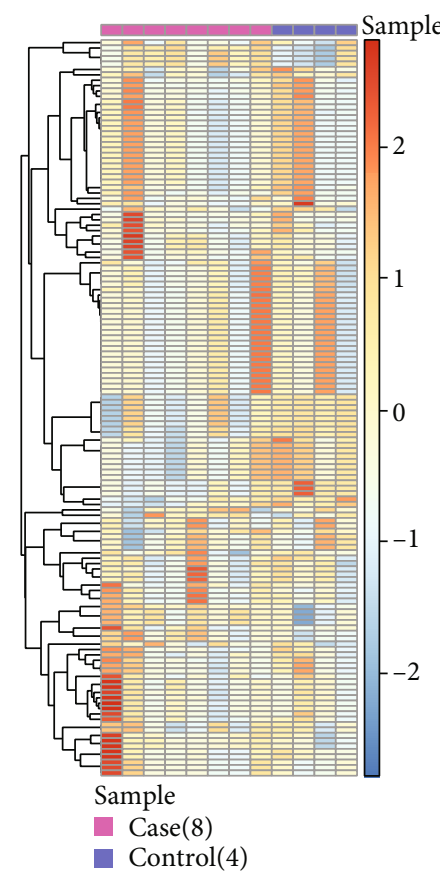

(d) GSE79705

FIGURE 9: Heatmaps depicting 137 signaling pathways enriched in the crosstalk genes in the four periodontitis-related datasets.

$\beta$-mediated apoptosis and found to be increased in the hippocampal regions of $\mathrm{AD}$ patients [61]. Myocyte-specific enhancer factor 2C (MEF2C) was identified as a critical transcription factor involved in the coexpression network of chronic periodontitis [62]. Genome-wide association studies (GWAS) have shown the linkage between mutation of MEF2C and aging-associated late-onset Alzheimer's disease $[63,64]$. Experimentally, a lack of MEF2C expression was shown to exaggerate microglial response and negatively affect brain function [65]. The USF2 (upstream transcription
TABLE 8: The number of crosstalk gene-related DEPs within the four periodontitis-related datasets.

\begin{tabular}{lcccccc}
\hline Data & Sample & $\begin{array}{c}\text { DEP } \\
\text { Up }\end{array}$ & $\begin{array}{c}\text { DEP } \\
\text { Down }\end{array}$ & $\begin{array}{c}\text { DEP } \\
\text { Total }\end{array}$ & $\begin{array}{c}\text { Log FC } \\
\text { Abs }\end{array}$ & $p$ value \\
\hline GSE23586 & 6 & 1 & 12 & 13 & $>1$ & $<0.05$ \\
GSE16134 & 310 & 84 & 2 & 86 & $>1$ & $<0.05$ \\
GSE10334 & 247 & 77 & 2 & 79 & $>1$ & $<0.05$ \\
GSE79705 & 12 & 4 & 4 & 8 & $>0$ & $<0.05$ \\
\hline
\end{tabular}




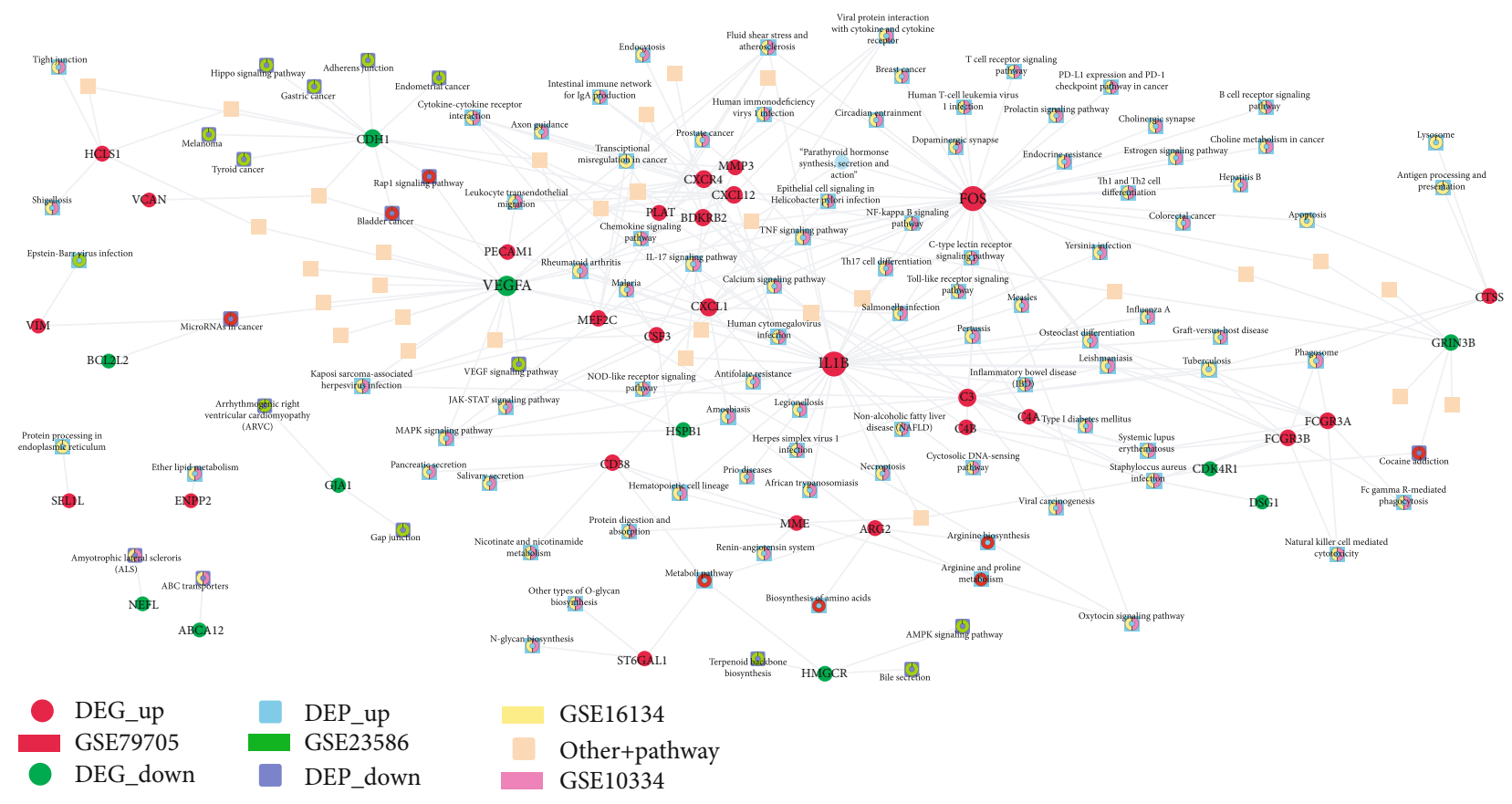

FIGURE 10: The crosstalk gene-differentially expressed pathway interaction network.

TABLE 9: The 12 overlapping genes between 48 feature selection-obtained crosstalk genes and periodontitis-related genes in the DisGeNET database. Among the 12 genes, 6 genes, C4A, C4B, CXCL12, FCGR3A, IL1B, and MMP3, were associated with chronic periodontitis.

\begin{tabular}{|c|c|c|c|}
\hline Gene symbol & Gene ID & Disease ID & Disease name \\
\hline $\mathrm{C} 3$ & 718 & C0031099 & Periodontitis \\
\hline $\mathrm{C} 4 \mathrm{~A}$ & 720 & C0266929 & Chronic periodontitis \\
\hline $\mathrm{C} 4 \mathrm{~B}$ & 721 & C0266929 & Chronic periodontitis \\
\hline CXCL12 & 6387 & C0266929 & Chronic periodontitis \\
\hline FCGR3A & 2214 & C0266929 & Chronic periodontitis \\
\hline FCGR3A & 2214 & C0031099 & Periodontitis \\
\hline FCGR3A & 2214 & C0031106 & Periodontitis, juvenile \\
\hline FCGR3A & 2214 & C0399447 & Early-onset periodontitis \\
\hline FCGR3A & 2214 & $\mathrm{C} 0031030$ & Periapical periodontitis \\
\hline FCGR3B & 2215 & C0399447 & Early-onset periodontitis \\
\hline FCGR3B & 2215 & C0031106 & Periodontitis, juvenile \\
\hline HSPB1 & 3315 & C0031030 & Periapical periodontitis \\
\hline IL1B & 3553 & C1719494 & Periodontitis, localized aggressive \\
\hline IL1B & 3553 & C1719495 & Aggressive periodontitis, generalized \\
\hline IL1B & 3553 & C0399447 & Early-onset periodontitis \\
\hline IL1B & 3553 & C0001342 & Acute periodontitis \\
\hline IL1B & 3553 & C0031106 & Periodontitis, juvenile \\
\hline IL1B & 3553 & C0031030 & Periapical periodontitis \\
\hline IL1B & 3553 & C4025886 & Severe periodontitis \\
\hline IL1B & 3553 & C0031099 & Periodontitis \\
\hline IL1B & 3553 & C0266929 & Chronic periodontitis \\
\hline MME & 4311 & C0031099 & Periodontitis \\
\hline MMP3 & 4314 & C0031106 & Periodontitis, juvenile \\
\hline MMP3 & 4314 & C0001342 & Acute periodontitis \\
\hline MMP3 & 4314 & C0266929 & Chronic periodontitis \\
\hline MMP3 & 4314 & C0031099 & Periodontitis \\
\hline PLAT & 5327 & C1719495 & Aggressive periodontitis, generalized \\
\hline VEGFA & 7422 & C0031099 & Periodontitis \\
\hline
\end{tabular}


ROC curve of gene expression value of chronic periodontitis

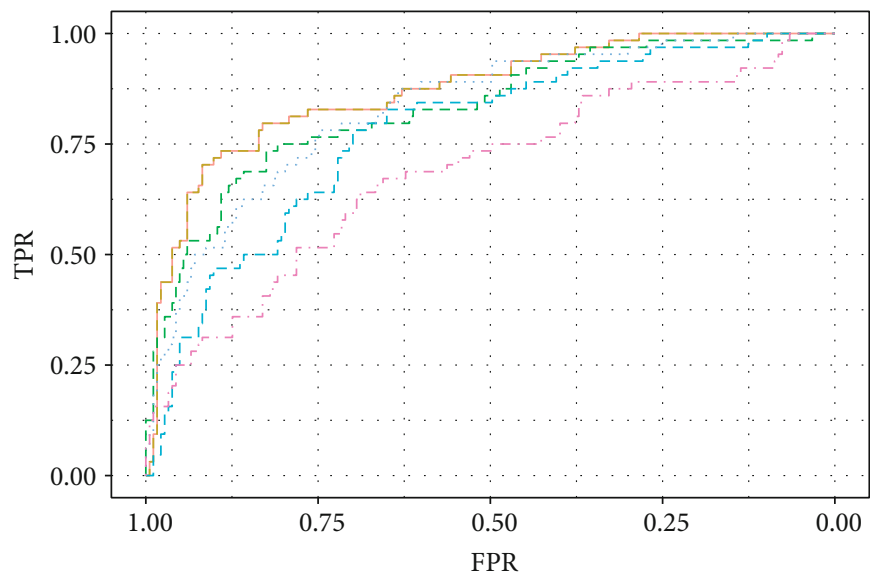

Name

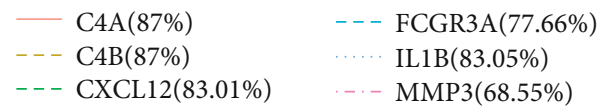

(a) GSE10334

ROC curve of gene expression value of chronic periodontitis

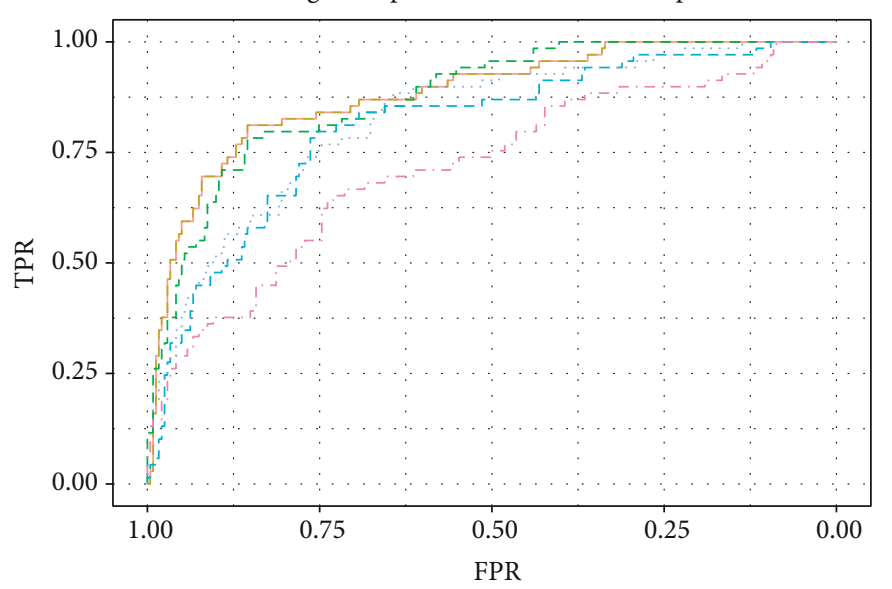

Name

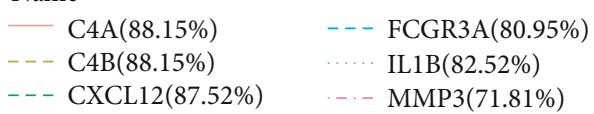

(b) GSE16134

Figure 11: Continued. 


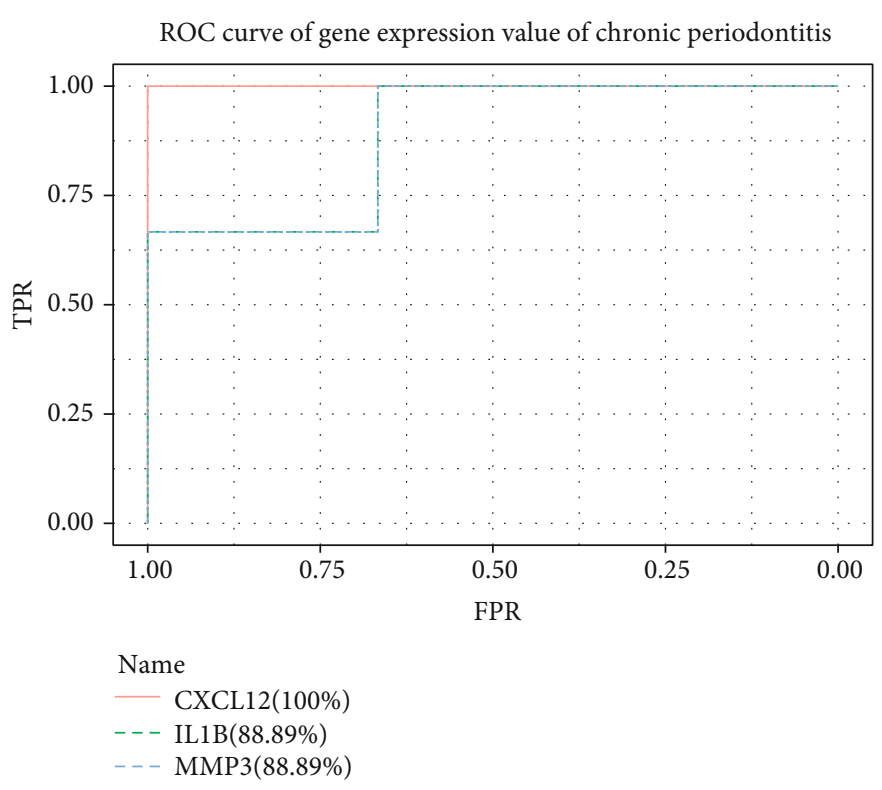

(c) GSE23586

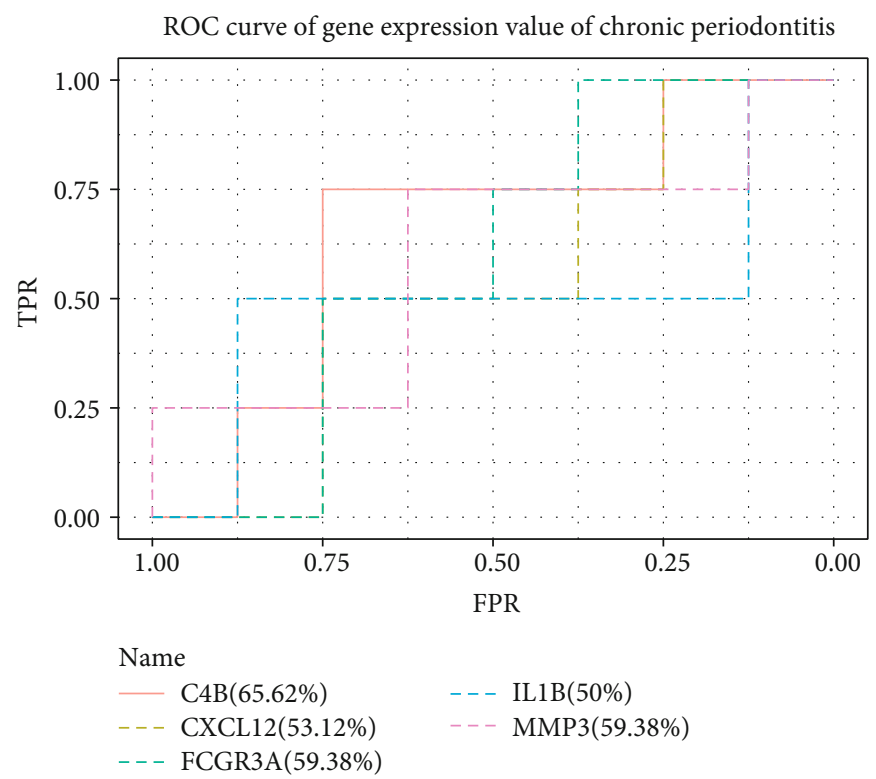

(d) GSE79705

Figure 11: The ROC curves for 6 chronic periodontitis-related genes, C4A, C4B, CXCL12, FCGR3A, IL1B, and MMP3, in the four periodontitis-related datasets.

factor 2, C-Fos interacting) transcription factor is reported to enhance osteogenic differentiation of periodontal ligament cells (PDLCs) [66]; however, its involvement in periodontal inflammation has not been reported. In the context of AD, the USF2 gene was shown to regulate the expression of genes Dhcr24, Aplp2, Tia1, Pdrx1, Vdac1, and Syn2, which drive the neuropathological mechanisms [67]. However, a study of Japanese participants found that the single nucleotide polymorphisms of the USF2 gene were not significantly related to the onset of $\mathrm{AD}$ [68].

Differentially expressed pathways were identified from the crosstalk gene-pathway network, and several pathways including JAK-STAT, MAPK, NF-kappa B, and natural killer cell-mediated cytotoxicity were found as the most robust differentially expressed pathways in at least two periodontitis datasets. Overall, experimental evidence supports these as linkage mechanisms between periodontitis and AD. The activation of the JAK-STAT pathway induced by the Porphyromonas gingivalis lipopolysaccharide (LPS) and nicotine was shown to increase the expression of cyclooxygenase-2 (COX-2), prostaglandin E2 (PGE2), and proinflammatory cytokines in osteoblasts, thus further accelerating periodontitis progression $[69,70]$. In $\mathrm{AD}$, the inhibitor of the JAKSTAK pathway is reported as a therapeutic target, and 
blocking this pathway can protect against neuroinflammation and dopaminergic neurodegeneration [71]. The MAPK pathway is the upstream signaling intermediate to many inflammatory cytokines such as TNF- $\alpha$, IL- $1 \beta$, IL-6, and prostaglandin E2 [72], and the blockage of this pathway could be beneficial for treating inflammatory diseases like chronic periodontitis and $\mathrm{AD}[73,74]$. The activation of MAPK signaling is noted to promote the production of MMPs and RANKL, leading to osteoclastogenesis and the acceleration of alveolar bone loss [73]. MAPK signaling is also implicated in multiple aspects of the neuropathology of $\mathrm{AD}$, such as promoting neuroinflammation, amyloid-beta toxicity and aggregation, autophagy, and apoptosis [75]. The overexpression of NF- $\kappa \mathrm{B}$ signaling plays a pivotal role in periodontitis-associated bone destruction by promoting the differentiation and activation of osteoclasts [76]. The blockade of NF- $\kappa \mathrm{B}$ signaling is found to trigger detrimental neural alterations including neuroinflammation, activation of microglia, oxidative stress-related complications, and apoptosis [77]. Natural killer (NK) cells are important regulators of innate and adaptive immunity and are closely linked to the regulation of cytotoxicity [78]. Experimental data shows NK cells can directly recognize the Fusobacterium nucleatum pathogen, leading to alveolar bone resorption and periodontitis [79]. The overactivity of NK is also purported to play a driving role in the progression of $\mathrm{AD}$ by producing a series of proinflammatory cytokines [80].

The findings of this in silico analysis must be considered in light of the strengths and limitations of this work. By using a machine learning-based feature selection method as the core technique, the most putatively robust crosstalk genes could be identified. Furthermore, functional molecular links were also analyzed in terms of differentially expressed pathways. The integrated analysis of multiple periodontitisrelated GEO datasets enabled a larger sample size for improved accuracy of our computational prediction in the present study. The major limitation of the current approach is that no experimental validation of the identified pivotal genetic molecular linkage candidates was performed. This work has multiple implications for future research. Experimental and clinical studies focused on these candidates could be valuable from the perspectives of identification of shared susceptibility, exaggerating pathogenic mechanisms, biomarkers, and therapeutic targets relevant to precision medicine and drug development or repurposing.

\section{Conclusion}

Bioinformatic analysis integrating experimental transcriptomic data from Alzheimer's disease and periodontitis revealed the most robust potentially shared molecular linkages. Six crosstalk genes, C4A, C4B, CXCL12, FCGR3A, IL1B, and MMP3, three transcription factors, FOS, MEF2C, and USF2, and several pathways, JAK-STAT, MAPK, NF-kappa B, and natural killer cell-mediated cytotoxicity, emerged as top candidate shared molecular linkage entities and merit future research in experimental and clinical studies.

\section{Data Availability}

The data used to support the findings of this study are available from the corresponding author upon request.

\section{Ethical Approval}

As this study only applied bioinformatic techniques based on computational analyses of publicly available datasets, therefore, this study did not require ethical approval.

\section{Consent}

Consent for publication is not applicable in this study because no individual person's data was used.

\section{Conflicts of Interest}

The authors declare no potential conflict of interest with respect to the authorship and publication of this paper.

\section{Authors' Contributions}

Lei Liu (Email: law1984@126.com), Baohua Xu (Email: zrkqxbh@163.com), and Xiaofeng Huang (Email: huangxf1998@163.com) contributed equally to this work as the corresponding author. Jieqi Jin (Email:jjq007@126.com), Mengkai Guang (Email: tygmk@qq.com), and Anthony Chukwunonso Ogbuehi (Email: treasurerite@yahoo.com) contributed equally as the first author. Dirk Ziebolz (Email: dirk.ziebolz@medizin.uni-leipzig.de) and Gerhard Schmalz (Email: gerhard.schmalz@medizin.uni-leipzig.de) contributed equally as the senior author.

\section{References}

[1] T. L. Cerajewska, M. Davies, and N. X. West, "Periodontitis: a potential risk factor for Alzheimer's disease," BDJ Team, vol. 3, no. 4, p. 16062, 2016.

[2] F. B. Teixeira, M. T. Saito, F. C. Matheus et al., "Periodontitis and Alzheimer's disease: a possible comorbidity between oral chronic inflammatory condition and neuroinflammation," Frontiers in Aging Neuroscience, vol. 9, p. 327, 2017.

[3] K. Hensley, "Neuroinflammation in Alzheimer's disease: mechanisms, pathologic consequences, and potential for therapeutic manipulation," Journal of Alzheimer's Disease, vol. 21, no. 1, pp. 1-14, 2010.

[4] D. F. Kinane, P. G. Stathopoulou, and P. N. Papapanou, "Periodontal diseases," Nature Reviews. Disease Primers, vol. 3, no. 1, p. 17038, 2017.

[5] J. Meyle and I. Chapple, "Molecular aspects of the pathogenesis of periodontitis," Periodontology 2000, vol. 69, no. 1, pp. 717, 2015.

[6] K. Abbayya, N. Y. Puthanakar, S. Naduwinmani, and Y. Chidambar, "Association between periodontitis and Alzheimer's disease," North American Journal of Medical Sciences, vol. 7, no. 6, pp. 241-246, 2015.

[7] S. S. Dominy, C. Lynch, F. Ermini et al., "Porphyromonas gingivalisin Alzheimer's disease brains: Evidence for disease causation and treatment with small-molecule inhibitors," Science Advances, vol. 5, no. 1, article eaau3333, 2019. 
[8] S. K. Singhrao, A. Harding, S. Poole, L. Kesavalu, and S. Crean, "Porphyromonas gingivalis periodontal infection and its putative links with Alzheimer's disease," Mediators of Inflammation, vol. 2015, Article ID 137357, 10 pages, 2015.

[9] L. M. Bekris, C.-E. Yu, T. D. Bird, and D. W. Tsuang, "Genetics of Alzheimer disease," Journal of Geriatric Psychiatry and Neurology, vol. 23, no. 4, pp. 213-227, 2010.

[10] G. Kaarthikeyan and S. Meenakshi, "Genetic biomarkers in periodontal disease diagnosis," in Periodontal DiseaseDiagnose Considerations, IntechOpen, 2019.

[11] M. S. Tonetti, H. Greenwell, and K. S. Kornman, "Staging and grading of periodontitis: framework and proposal of a new classification and case definition," Journal of Clinical Periodontology, vol. 45, Supplement 20, pp. S149-S161, 2018.

[12] M. E. Ritchie, B. Phipson, Y. H. Di Wu, C. W. Law, W. Shi, and G. K. Smyth, "limma powers differential expression analyses for RNA-sequencing and microarray studies," Nucleic Acids Research, vol. 43, no. 7, article e47, 2015.

[13] S. Li, X. Chen, X. Liu et al., "Complex integrated analysis of lncRNAs-miRNAs-mRNAs in oral squamous cell carcinoma," Oral Oncology, vol. 73, pp. 1-9, 2017.

[14] G. Dennis, B. T. Sherman, D. A. Hosack et al., "DAVID: database for annotation, visualization, and integrated discovery," Genome Biology, vol. 4, no. 9, pp. 1-11, 2003.

[15] J. Bai, Y. Li, T. Shao et al., "Integrating analysis reveals microRNA-mediated pathway crosstalk among Crohn's disease, ulcerative colitis and colorectal cancer," Molecular BioSystems, vol. 10, no. 9, pp. 2317-2328, 2014.

[16] S. Li, X. Liu, H. Li et al., "Integrated analysis of long noncoding RNA-associated competing endogenous RNA network in periodontitis," Journal of Periodontal Research, vol. 53, no. 4, pp. 495-505, 2018.

[17] Y. Dong, Y. Xiao, Q. Shi, and C. Jiang, "Dysregulated lncRNAmiRNA-mRNA network reveals patient survival-associated modules and RNA binding proteins in invasive breast carcinoma," Frontiers in Genetics, vol. 10, p. 1284, 2020.

[18] T. S. K. Prasad, R. Goel, K. Kandasamy et al., "Human protein reference database-2009 update," Nucleic Acids Research, vol. 37, Supplement 1, pp. D767-D772, 2009.

[19] R. Oughtred, J. Rust, C. Chang et al., “The BioGRID database: a comprehensive biomedical resource of curated protein, genetic and chemical interactions," Protein Science, vol. 30, pp. 187-200, 2021.

[20] I. Xenarios, D. W. Rice, L. Salwinski, M. K. Baron, E. M. Marcotte, and D. Eisenberg, "DIP: the database of interacting proteins," Nucleic Acids Research, vol. 28, no. 1, pp. 289-291, 2000.

[21] A. Zanzoni, L. Montecchi-Palazzi, M. Quondam, G. Ausiello, M. Helmer-Citterich, and G. Cesareni, "MINT: a Molecular INTeraction database," FEBS Letters, vol. 513, no. 1, pp. 135140, 2002.

[22] M. J. Cowley, M. Pinese, K. S. Kassahn et al., "PINA v2. 0: mining interactome modules," Nucleic Acids Research, vol. 40, no. D1, pp. D862-D865, 2012.

[23] K. Breuer, A. K. Foroushani, M. R. Laird et al., "InnateDB: systems biology of innate immunity and beyond-recent updates and continuing curation," Nucleic Acids Research, vol. 41, no. D1, pp. D1228-D1233, 2013.

[24] M. J. Meyer, J. Das, X. Wang, and H. Yu, "INstruct: a database of high-quality $3 \mathrm{D}$ structurally resolved protein interactome networks," Bioinformatics, vol. 29, no. 12, pp. 1577-1579, 2013.
[25] Y. Li, J. Xu, H. Chen et al., "Characterizing genes with distinct methylation patterns in the context of protein-protein interaction network: application to human brain tissues," PLoS One, vol. 8, no. 6, article e65871, 2013.

[26] J. Piñero, À. Bravo, N. Queralt-Rosinach et al., "DisGeNET: a comprehensive platform integrating information on human disease-associated genes and variants," Nucleic Acids Research, vol. 45, no. D1, pp. D833-D839, 2017.

[27] M. B. Kursa and W. R. Rudnicki, "Feature selection with the Boruta package," Journal of Statistical Software, vol. 36, no. 11, pp. 1-13, 2010.

[28] H. Sanz, C. Valim, E. Vegas, J. M. Oller, and F. Reverter, "SVM-RFE: selection and visualization of the most relevant features through non-linear kernels," BMC Bioinformatics, vol. 19, no. 1, pp. 1-18, 2018.

[29] L. Torgo, Data Mining with R: Learning with Case Studies, Chapman \& Hall/CRC Data Mining and Knowledge Discovery Series, 2010.

[30] F. Pedregosa, G. Varoquaux, A. Gramfort et al., "Scikit-learn: machine learning in Python," The Journal of Machine Learning Research, vol. 12, pp. 2825-2830, 2011.

[31] M. Qiu, Q. Fu, C. Jiang, and D. Liu, "Machine learning based network analysis determined clinically relevant miRNAs in breast cancer," Frontiers in Genetics, vol. 11, p. 1467, 2020.

[32] H. Han, J.-W. Cho, S. Lee et al., "TRRUST v2: an expanded reference database of human and mouse transcriptional regulatory interactions," Nucleic Acids Research, vol. 46, no. D1, pp. D380-D386, 2018.

[33] H. Xu, H. Yu, K. Tu et al., “cGRNB: a web server for building combinatorial gene regulatory networks through integrated engineering of seed-matching sequence information and gene expression datasets," BMC Systems Biology, vol. 7, no. S2, p. S7, 2013.

[34] L. A. Bovolenta, M. L. Acencio, and N. Lemke, "HTRIdb: an open-access database for experimentally verified human transcriptional regulation interactions," BMC Genomics, vol. 13, no. 1, p. 405, 2012.

[35] F. Vafaee, J. R. Krycer, X. Ma, T. Burykin, D. E. James, and Z. Kuncic, "ORTI: an open-access repository of transcriptional interactions for interrogating mammalian gene expression data," PLoS One, vol. 11, no. 10, article e0164535, 2016.

[36] E. Wingender, P. Dietze, H. Karas, and R. Knüppel, “TRANSFAC: a database on transcription factors and their DNA binding sites," Nucleic Acids Research, vol. 24, no. 1, pp. 238-241, 1996.

[37] Y. Li, T. Shao, C. Jiang et al., "Construction and analysis of dynamic transcription factor regulatory networks in the progression of glioma," Scientific Reports, vol. 5, no. 1, pp. 1-12, 2015.

[38] M. Seppänen, M. L. Lokki, I. L. Notkola et al., "Complement and c4 null alleles in severe chronic adult periodontitis," Scandinavian Journal of Immunology, vol. 65, no. 2, pp. 176-181, 2007.

[39] D. G. Walker and P. L. McGeer, "Complement gene expression in human brain: comparison between normal and Alzheimer disease cases," Molecular Brain Research, vol. 14, no. 1-2, pp. 109-116, 1992.

[40] A. M. Havens, E. Chiu, M. Taba Jr. et al., "Stromal-derived factor- $1 \alpha$ (CXCL12) levels increase in periodontal disease," Journal of Periodontology, vol. 79, no. 5, pp. 845-853, 2008. 
[41] A. Parachikova and C. Cotman, "Reduced CXCL12/CXCR4 results in impaired learning and is downregulated in a mouse model of Alzheimer disease," Neurobiology of Disease, vol. 28, no. 2, pp. 143-153, 2007.

[42] G. G. Song and Y. H. Lee, "Associations between FCGR2A rs1801274, FCGR3A rs396991, FCGR3B NA1/NA2 polymorphisms and periodontitis: a meta-analysis," Molecular Biology Reports, vol. 40, no. 8, pp. 4985-4993, 2013.

[43] J. P. Fuller, J. B. Stavenhagen, and J. L. Teeling, "New roles for Fc receptors in neurodegeneration-the impact on immunotherapy for Alzheimer's disease," Frontiers in Neuroscience, vol. 8, p. 235, 2014.

[44] R. Cheng, Z. Wu, M. Li, M. Shao, and T. Hu, "Interleukin- $1 \beta$ is a potential therapeutic target for periodontitis: a narrative review," International Journal of Oral Science, vol. 12, no. 1, pp. 1-9, 2020.

[45] S. L. M. Payão, G. M. Gonçalves, R. W. de Labio et al., "Association of interleukin $1 \beta$ polymorphisms and haplotypes with Alzheimer's disease," Journal of Neuroimmunology, vol. 247, no. 1-2, pp. 59-62, 2012.

[46] C. Franco, H.-R. Patricia, S. Timo, B. Claudia, and H. Marcela, "Matrix metalloproteinases as regulators of periodontal inflammation," International Journal of Molecular Sciences, vol. 18, no. 2, p. 440, 2017.

[47] X.-X. Wang, M.-S. Tan, J.-T. Yu, and L. Tan, "Matrix metalloproteinases and their multiple roles in Alzheimer's disease," BioMed Research International, vol. 2014, Article ID 908636, 8 pages, 2014.

[48] C. Holmes, "Review: systemic inflammation and Alzheimer's disease," Neuropathology and Applied Neurobiology, vol. 39, no. 1, pp. 51-68, 2013.

[49] V. H. Perry and J. Teeling, "Microglia and macrophages of the central nervous system: the contribution of microglia priming and systemic inflammation to chronic neurodegeneration," in Seminars in immunopathology, Springer, 2013.

[50] G. Ramesh, A. G. MacLean, and M. T. Philipp, "Cytokines and chemokines at the crossroads of neuroinflammation, neurodegeneration, and neuropathic pain," Mediators of Inflammation, vol. 2013, Article ID 480739, 20 pages, 2013.

[51] G. Hajishengallis, T. Maekawa, T. Abe, E. Hajishengallis, and J. D. Lambris, "Complement involvement in periodontitis: molecular mechanisms and rational therapeutic approaches," Immune Responses to Biosurfaces, vol. 865, pp. 57-74, 2015.

[52] E. V. Clarke and A. J. Tenner, "Complement modulation of T cell immune responses during homeostasis and disease," Journal of Leukocyte Biology, vol. 96, no. 5, pp. 745-756, 2014.

[53] K. W-h, W. van der Touw, and P. S. Heeger, "Complement regulation of $\mathrm{T}$ cell immunity," Immunologic Research, vol. 54, no. 1-3, pp. 247-253, 2012.

[54] A. Guyon, "CXCL12 chemokine and its receptors as major players in the interactions between immune and nervous systems," Frontiers in Cellular Neuroscience, vol. 8, p. 65, 2014.

[55] R. Ransohoff, "The chemokine system in neuroinflammation: an update," The Journal of Infectious Diseases, vol. 186, Supplement 2, pp. S152-S1S6, 2002.

[56] W. H. Yeap, K. L. Wong, N. Shimasaki et al., "CD16 is indispensable for antibody-dependent cellular cytotoxicity by human monocytes," Scientific Reports, vol. 6, no. 1, p. 34310, 2016.

[57] S. Solerte, M. Fioravanti, A. Pascale, E. Ferrari, S. Govoni, and F. Battaini, "Increased natural killer cell cytotoxicity in Alzhei- mer's disease may involve protein kinase C dysregulation," Neurobiology of Aging, vol. 19, no. 3, pp. 191-199, 1998.

[58] S. S. Shaftel, W. S. T. Griffin, and M. K. O'Banion, “The role of interleukin-1 in neuroinflammation and Alzheimer disease: an evolving perspective," Journal of Neuroinflammation, vol. 5, no. 1, pp. 1-12, 2008.

[59] E. M. Kim and O. Hwang, "Role of matrix metalloproteinase-3 in neurodegeneration," Journal of Neurochemistry, vol. 116, no. 1, pp. 22-32, 2011.

[60] A. Trabandt, R. Gay, V. Sukhatme, and S. Gay, "Expression of collagenase and potential transcriptional factors c-fos and egr1 in periodontal gingival fibroblasts," Journal of Oral Pathology \& Medicine, vol. 21, no. 5, pp. 232-240, 1992.

[61] D. L. Marcus, J. A. Strafaci, D. C. Miller et al., "Quantitative neuronal c-fos and c-jun expression in Alzheimer's disease," Neurobiology of Aging, vol. 19, no. 5, pp. 393-400, 1998.

[62] G. Sun, T. Jiang, P. Xie, and J. Lan, "Identification of the disease-associated genes in periodontitis using the coexpression network," Molecular Biology, vol. 50, no. 1, pp. 124-131, 2016.

[63] S. L. Rosenthal and M. I. Kamboh, "Late-onset Alzheimer's disease genes and the potentially implicated pathways," Current Genetic Medicine Reports, vol. 2, no. 2, pp. 85-101, 2014.

[64] J.-C. Lambert, European Alzheimer's Disease Initiative (EADI), C. A. Ibrahim-Verbaas et al., "Meta-analysis of 74,046 individuals identifies 11 new susceptibility loci for Alzheimer's disease," Nature Genetics, vol. 45, no. 12, pp. $1452-$ 1458, 2013.

[65] A. Deczkowska, O. Matcovitch-Natan, A. Tsitsou-Kampeli et al., "Mef2C restrains microglial inflammatory response and is lost in brain ageing in an IFN-I-dependent manner," Nature Communications, vol. 8, no. 1, pp. 1-13, 2017.

[66] F. Liu, X. Wang, B. Zheng, D. Li, and C. Chen, "USF2 enhances the osteogenic differentiation of PDLCs by promoting ATF4 transcriptional activities," Journal of Periodontal Research, vol. 55, no. 1, pp. 68-76, 2020.

[67] G. K. Acquaah-Mensah and R. C. Taylor, "Brain in situ hybridization maps as a source for reverse-engineering transcriptional regulatory networks: Alzheimer's disease insights," Gene, vol. 586, no. 1, pp. 77-86, 2016.

[68] N. Shibata, T. Ohnuma, S. Higashi et al., "Genetic association between USF 1 and USF 2 gene polymorphisms and Japanese Alzheimer's disease," The Journals of Gerontology Series A: Biological Sciences and Medical Sciences, vol. 61, no. 7, pp. 660662, 2006.

[69] H. Y-k and I. S. Lee, "JAK/STAT pathway modulates on Porphyromonas gingivalis lipopolysaccharide-and nicotineinduced inflammation in osteoblasts," Journal of Dental Hygiene Science, vol. 17, no. 1, pp. 81-86, 2017.

[70] W. Wei, X. Xiao, J. Li et al., "Activation of the STAT1 pathway accelerates periodontitis in Nos3-/- mice," Journal of Dental Research, vol. 98, no. 9, pp. 1027-1036, 2019.

[71] H. Qin, J. A. Buckley, X. Li et al., "Inhibition of the JAK/STAT pathway protects against $\alpha$-synuclein-induced neuroinflammation and dopaminergic neurodegeneration," Journal of Neuroscience, vol. 36, no. 18, pp. 5144-5159, 2016.

[72] H. Chi, S. P. Barry, R. J. Roth et al., "Dynamic regulation of pro- and anti-inflammatory cytokines by MAPK phosphatase 1 (MKP-1) in innate immune responses," Proceedings of the National Academy of Sciences, vol. 103, no. 7, pp. 2274-2279, 2006. 
[73] K. L. Kirkwood and C. Rossa Jr., "The potential of p38 MAPK inhibitors to modulate periodontal infections," Current Drug Metabolism, vol. 10, no. 1, pp. 55-67, 2009.

[74] L. Munoz and A. J. Ammit, "Targeting p38 MAPK pathway for the treatment of Alzheimer's disease," Neuropharmacology, vol. 58, no. 3, pp. 561-568, 2010.

[75] G. Kheiri, M. Dolatshahi, F. Rahmani, and N. Rezaei, "Role of p38/MAPKs in Alzheimer's disease: implications for amyloid beta toxicity targeted therapy," Reviews in the Neurosciences, vol. 30, no. 1, pp. 9-30, 2018.

[76] R. Ambili and P. Janam, “A critique on nuclear factor-kappa B and signal transducer and activator of transcription 3: the key transcription factors in periodontal pathogenesis," Journal of Indian Society of Periodontology, vol. 21, no. 5, pp. 350-356, 2017.

[77] N. K. Jha, S. K. Jha, R. Kar, P. Nand, K. Swati, and V. K. Goswami, "Nuclear factor-kappa $\beta$ as a therapeutic target for Alzheimer's disease," Journal of Neurochemistry, vol. 150, no. 2, pp. 113-137, 2019.

[78] L. Parisi, B. Bassani, M. Tremolati, E. Gini, G. Farronato, and A. Bruno, "Natural killer cells in the orchestration of chronic inflammatory diseases," Journal of Immunology Research, vol. 2017, Article ID 4218254, 13 pages, 2017.

[79] S. Chaushu, A. Wilensky, C. Gur et al., "Direct recognition of Fusobacterium nucleatum by the NK cell natural cytotoxicity receptor NKp46 aggravates periodontal disease," PLoS Pathogens, vol. 8, no. 3, article e1002601, 2012.

[80] F. Jadidi-Niaragh, H. Shegarfi, F. Naddafi, and A. Mirshafiey, "The role of natural killer cells in Alzheimer's disease," Scandinavian Journal of Immunology, vol. 76, no. 5, pp. 451-456, 2012. 